\title{
GAUGE EFFECTS ON PHASE TRANSITIONS IN SUPERCONDUCTORS
}

\author{
D. V. Shopova ${ }^{1 *}$, T. E. Tsvetkov ${ }^{1}$, D. I. Uzunov ${ }^{1,2}$ \\ ${ }^{1}$ CP Laboratory, Institute of Solid State Physics, \\ Bulgarian Academy of Sciences, BG-1784 Sofia, Bulgaria, \\ ${ }^{2}$ Max-Plank-Institut für Physik komplexer Systeme, Nötnitzer Str. 38, 01187 Dresden, Germany, \\ *Corresponding author: sho@issp.bas.bg
}

(Received October 02, 2006; in final form - April 16, 2007)

\begin{abstract}
Classic and recent results for gauge effects on the properties of the normal-to-superconducting phase transition in bulk and thin film superconductors are reviewed. Similar problems in the description of other natural systems (liquid crystals, quantum field theory, early Universe) are also discussed. The relatively strong gauge effects on the fluctuations of the ordering field at low spatial dimensionality $D$ and, in particular, in thin (quasi-2D) films are considered in detail. Special attention is paid to the fluctuations of the gauge field. It is shown that the mechanism in which these gauge fluctuations affect the order of the phase transition and other phase transition properties varies with the variation of the spatial dimensionality $D$. The problem for the experimental confirmation of the theoretical predictions about the order of the phase transitions in gauge systems is discussed.
\end{abstract}

Key words: thermodynamics, order parameter, specific heat, equation of state, renormalization group, fluctuation.

PACS number(s): 74.20.De, 74.78.Db

\section{INTRODUCTION}

\section{A. Superconductivity and gauge effects}

A remarkable example of gauge theory in condensed matter physics is the Ginzburg-Landau (GL) functional of superconductivity $[1,2]$. The latter is invariant towards both global $U(1)$ rotations of the order parameter field $\psi(\mathbf{x})$ and local gauge transformations (rotations) of the same field and the vector potential $\mathbf{A}(\mathbf{x})$ of the magnetic induction $\mathbf{B}$. These two properties of gauge invariance define the global and local $U(1)$ symmetries of the GL theory. In both cases the gauge group is a onedimensional Abelian continuous group $U(1)$. The spontaneous breaking of these global and local symmetries below the phase transition point allows for the appearance of the superconducting phases: the uniform Meissner phase and, under certain circumstances, the mixed (Abrikosov vortex [3]) phase. While the Meissner phase $[\langle\psi\rangle \neq 0,\langle\mathbf{B}\rangle=0]$ is a mere product of the breaking of the global $U(1)$ symmetry, the vortex phase $[\langle\psi(\mathbf{x})\rangle \neq 0$, $\langle\mathbf{B}(\mathbf{x})\rangle \neq 0$ ], where the equilibrium field configurations of both the order parameter field $\psi(\mathbf{x})$ and magnetic induction $\mathbf{B}(\mathbf{x})$ are spatially nonuniform, is a result of the spontaneous breaking of local $U(1)$ symmetry. The local gauge is important also for the description of the magnetic field penetration in both Meissner and vortex phases. This penetration is described by an additional characteristic length, the London penetration length $[1,2]$, which is different from zero only when the symmetry is broken, i. e., in the ordered phases, where the equilibrium value of $\psi$ is different from zero. The local $U(1)$ symmetry is present when the superconductor is an external magnetic field, or, when this field is equal to zero but magnetic fluctuations exist and give sense of the vector potential $\mathbf{A}(\mathbf{x})$ as a purely fluctuating field.

The fluctuations of the relevant fields, $\psi(\mathbf{x})$ and $\mathbf{A}(\mathbf{x})$ in usual superconductors are small and can be neglected. This means that the GL functional can be investigated in the lowest order mean field (MF) approximation (alias, "tree approximation" $[4,5]$ ). This is the usual way of treatment of the GL free energy, in particular, the investigation of the GL equations [1-3]. Within the tree approximation, the phase transition from normal to superconducting state in zero magnetic field is of the second order $[1,2,4]$. For a long time this phase transition has been considered as one of the best examples of the second order phase transitions, which has an excellent description within MF. But in 1974 Halperin, Lubensky and $\mathrm{Ma}$ (HLM) [6] showed that the magnetic fluctuations change the order of the superconducting phase transition in a zero external magnetic field $\left.\left(H_{0}=\left|\mathbf{H}_{0}\right|=0\right)\right)$, i. e., the order of the phase transition from normal-to-uniform (Meissner) superconducting state at $T_{c 0}=T_{c}\left(H_{0}=0\right.$ ) (see, also Ref. [7]). Since then this fluctuation change of the order of normal-to-superconducting phase transition (HLM effect) has been under debate. After 1974 an overwhelming amount of theoretical research on this topic has been performed but up to now there is no complete consensus about the order of this phase transition. In this review we shall consider some aspects of this problem but we shall not be able to discuss or mention all relevant contributions. However, it is important to emphasize that the investigation of the properties of the superconducting phase transition is important for other areas of physics, too. Here we shall briefly enumerate and discuss several examples. 


\section{B. Quantum field theory and other problems}

In elementary particle physics the gauge invariant theory similar to the GL theory of superconductivity is called the the Abelian Higgs Model [8-13]. The same global $U(1)$ and local $U(1)$ gauge symmetries are present but the phenomenon of spontaneous breaking of symmetry occurs only for imaginary mass of the Higgs $(\psi)$ field, which is an analog of the field $\psi$ describing the Cooper pair in a superconductor. In the absence of spontaneous symmetry breaking this model would describe an ordinary electrodynamics of charged scalars, but the situation becomes more interesting when the mentioned mass is imaginary and the breaking of symmetry occurs. Now the symmetry breaking phenomena receive other names and another physical interpretation. The breaking of both global and local gauge symmetries ensures a mechanism of transformation of the two initial scalar fields - analogs of the components $\psi^{\prime}$ and $\psi^{\prime \prime}$ of the complex field $\psi=\psi^{\prime}+i \psi^{\prime \prime}$, and two massless photon fields - analogs of the two independent components $A_{j}$ of the vector potential $\mathbf{A}=\left\{A_{j} ; j=1,2,3 ; \boldsymbol{\nabla} \cdot \mathbf{A}=0\right\}$ in a superconductor, to four massive particle fields: the so-called Higgs boson, which is an analog of the spontaneous order $|\psi|>0$ in a superconductor, and three massive vector field components, i. e. a massive three dimensional vector field. The mass of this new vector field is proportional to the electric charge $|q|$ and magnitude $|\psi|$ of the Higgs field and, as a matter of fact, this is exactly the way, in which the London penetration length in a superconductor depends on the electron charge $|e|$ and the modulus $|\psi|$ of the superconducting order parameter $\psi$. Thus the spontaneous breaking of the local gauge symmetry leads to the formation of massive particles without spoiling the gauge invariance of the theory. This is called "the Higgs mechanism". The latter plays a fundamental role in the unified theory of electromagnetic, weak, and strong interactions (see, e. g., Refs. [12,13]).

It is easy to see that there is something quite common between the phenomena of spontaneous breaking of the continuous symmetries in superconductivity theory and in quantum field theory. The superconducting phase $|\psi|>0$ is the exact analog of the Higgs boson in the Abelian-Higgs model, whereas the appearance of a massive vector field has its analog in the finite London penetration length mentioned above. Of course, there are no obstacles in interpreting the phenomena of spontaneous symmetry breaking in quantum field theory as phase transitions by taking the Higgs mode mass as a tuning parameter. The phase transition will occur at zero mass of the Higgs boson.

A similar phenomenon of spontaneous breaking of both global $U(1)$ and local gauge symmetries is possible also within the scalar electrodynamics due to mass insertions from the radiation corrections, as shown in Ref. [14]. The radiation corrections, analogs of the magnetic fluctuations in a superconductor, generate an imaginary mass to the initially massless scalar field in this theory and the latter becomes very like the Abelian-Higgs model. Here the symmetry breaking leads to the appear- ance of massive scalar and vector fields describing neutral scalar meson and vector meson, respectively [14]. The radiation corrections to the Lagrangian of the massless scalar electrodynamics [14] resembles very much, in particular, in their mathematical form at $D=4$, the magnetic fluctuation corrections to the GL free energy of 4D superconductors [7]. The interrelationships between the superconductivity theory and the gauge theories of elementary particles has been comprehensively discussed in Ref. [15]. Note also the interrelationship between the GL functional of superconductivity and the $C P^{N-1}$ confinement model (see Ref. [16]) and extensions to non-Abelian theories [17].

The gauge theories, mentioned so far, and their extensions have a wide application in the description of the Early Universe [15,18]. Another interesting gauge theory is that of the nematic-to-smectic $A$ phase transition in liquid crystals. According to the Kobayashi-McMillan-de Gennes theory [19-21] the smectic- $A$ order is described by two order parameters: the nematic director vector and the complex scalar describing the center of masses of the long molecules. When a description, quite analogous to that of superconductors, is introduced, as suggested by de Gennes [21], the director vector is substituted with a gauge vector field, which is quite similar to the vector potential A in the GL functional. Apart from some specific features intended to take into account the liquid crystal anisotropy, the effective free energy of the smectic A looks very like the GL free energy of superconductors (see, also, Refs. [22-24]). Another gauge theory in condensed matter physics that has some (but not very close) similarity with the GL free energy of superconductors is the Chern-Simons-Ginzburg-Landau (CSGL) effective functional of the quantum Hall liquid state, in particular, in the description of the phase transitions between the plateaus in the quantum Hall effect [25-28]. Let us mention also the liquid metallic hydrogen, where the problem of the superconducting-to-superfluid phase transition [29] is also related to the topics discussed in this review.

\section{About the investigation of the fluctuation-driven first order phase transition in superconductors}

Now we shall focus on the fluctuation effects for the properties of the normal-to-superconducting phase transition in the zero external magnetic field, which is the subject of the present review and in particular cases we shall refer to related topics in other natural systems. We shall consider in more detail the HLM effect in 2D and quasi-2D superconductors with a special emphasis on the problem of the theoretical predictions reliability. Following Ref. [6] we shall use two theoretical methods.

In Sec. II we use the MF like approximation of Ref. [6] where the spatial fluctuations of superconducting order parameter $\psi$ are neglected, and the magnetic fluctuations are taken into consideration. This treatment was justified for the well established type I superconductors, where the London penetration length $\lambda$ is much smaller 
than the coherence (correlation) length $\xi$. Due to the neglecting of spatial fluctuations of $\psi$, the results of such MF treatment should be valid outside the Ginzburg critical region [4]. A weakly first order phase transition occurs as a result of a new small $|\psi|^{3}$-term, which appears in the effective free energy of $3 \mathrm{D}$ superconductors within the framework of the MF like approximation [6]. But this HLM effect was found to be very small and experimentally unobservable even for the well established type I bulk (3D-) superconductors, such as Al, where the GL number $\kappa=\lambda / \xi=10^{-2} \ll 1$. It has been recently shown $[30-36]$ that the HLM effect is much stronger in quasi-2D superconductors than in the bulk (3D) samples. Moreover, as shown in this series of papers, the effect appears by a term of type $|\psi|^{2} \ln |\psi|$ in the effective free energy, and essentially depends on the thickness $L_{0}$ of quasi-2D superconducting films. These circumstances provide a real opportunity for an experimental verification of the effect - a topic of discussion throughout the present review, and in particular, in Sec. II.

We must emphasize that MF results are not valid for thermodynamic states in the Ginzburg critical region $(\delta T)_{G}=\left|T_{G}-T_{c 0}\right| / T_{c 0}$ around the equilibrium phase transition point $T_{c 0}$. In certain classes of hightemperature superconductors the Ginzburg region exceeds $0.1 \mathrm{~K}$ whereas in usual low-temperature superconductors it is very narrow, $(\delta T)_{G} \sim 10^{-12}-10^{-16} \mathrm{~K}$, and the respective critical states are experimentally unaccessible (see, e.g., [4]). If the metastability states, which go along with the weakly first order phase transition predicted by MF, extend over the temperature intervals larger than the size of the Ginzburg region, one may conclude that the MF prediction of the HLM effect is reliable. In Sec. II we justify the MF analysis reliability for such element superconductors as Al, W, In. We will show that the condition for sufficient wideness of the metastability regions is quite strong and, perhaps, irrelevant in experiments. We will also demonstrate that the magnitude of the critical magnetic field is crucial for the observability of the HLM effect in thin superconducting films (see also the discussion in Sec. II.I).

In Sec. III we review some results for the HLM effect obtained with the help of the renormalization group (RG) method $[4,5]$. The latter allows simultaneous treatment of both superconducting and magnetic fluctuations in the asymptotically close vicinity of the phase transition point. The lack of fixed point of the one-loop RG equations for conventional superconductors in zero external magnetic field was interpreted as a signal for a fluctuation-driven first order phase transition [6]. This result shows that the local gauge magnetic fluctuations are relevant also in the Ginzburg region of strong $\psi$ fluctuations and under certain circumstances they can change the order of the phase transition to a weakly first order, as is outside the critical region. The RG investigations of the superconducting phase transition order in zero magnetic field has been recently reviewed in Ref. [37]. While the latter review emphasizes the investigation of the HLM effect in high orders of the loop expansion $[4,5]$, here we lay stress on RG results for the effects of anisotropy, quenched disorder and extended symmetries of the Higgs $\psi$-field on the phase transition properties. The RG study of unconventional superconductors will also be discussed. In the remainder of this paper we shall consider mainly the phase transition to the superconducting state but some results for other systems and related topics will also be mentioned in brief; see Sec. III.C-III.E.

\section{MF STUDIES}

\section{A. General GL functional}

The GL free energy [2] of D-dimensional superconductor of volume $V_{D}=\left(L_{1} \ldots L_{D}\right)$ is given in the form

$$
\begin{aligned}
F(\psi, \mathbf{A}) & =\int d^{D} x\left[a|\psi|^{2}+\frac{b}{2}|\psi|^{4}\right. \\
& \left.+\frac{\hbar^{2}}{4 m}\left|\left(\nabla-\frac{2 i e}{\hbar c} \mathbf{A}\right) \psi\right|^{2}+\frac{\mathbf{B}^{2}}{8 \pi}\right] .
\end{aligned}
$$

In Eq. (1) the first Landau parameter $a=\alpha_{0}\left(T-T_{c 0}\right)$ is expressed by the critical temperature $T_{c 0}=T_{c}(H=0)$ in zero external magnetic field $(H=|\mathbf{H}|), b>0$ is the second Landau parameter and $e \equiv|e|$ is the electron charge. The square $\mathbf{B}^{2}$ of the magnetic induction $\mathbf{B}=(\mathbf{H}+4 \pi \mathbf{M})$, is given by the vector potential $\mathbf{A}(\mathbf{x})=\left\{A_{j}(\mathbf{x}), j=1, \ldots, D\right\}$ in the form

$$
\mathbf{B}^{2}=\frac{1}{2} \sum_{i, j=1}^{D}\left(\frac{\partial A_{j}}{\partial x_{i}}-\frac{\partial A_{i}}{\partial x_{j}}\right)^{2}
$$

here the vector potential $\mathbf{A}(\mathbf{x})$ obeys the Coulomb gauge $\nabla \cdot \mathbf{A}(\mathbf{x})=0$. For $3 \mathrm{D}$ superconductor the relation $\mathbf{B}=\boldsymbol{\nabla} \times \mathbf{A}(\mathbf{x})$ can be used and when $\mathbf{B}=\mathbf{B}_{0}$ is uniform along the $z$-axis, the Landau gauge $\mathbf{A}_{0}(\mathbf{x})=$ $B_{0}(-y / 2,-x / 2,0)$ can be applied. This representation can be generalized for the $(D>2)$-dimensional systems, where the magnetic induction $B_{0}$ is second rank tensor [38]:

$$
B_{0 i j}=B_{0}\left(\delta_{i 1} \delta_{j 2}-\delta_{j 2} \delta_{i 1}\right)
$$

If we use the notation $\mathbf{x}=\left(x_{1}, x_{2}, \mathbf{r}\right)$, where $\mathbf{r}$ is a $(D-2)$-dimensional vector, perpendicular to the plane $\left(x_{1}, x_{2}\right)$, in the $3 D$ case we will have $\mathbf{r}=(0,0, z)$, and

$$
B_{j}=\frac{1}{2} \epsilon_{j k l} B_{0 k l}=B_{0} \delta_{j 3},
$$

where $\epsilon_{j k l}$ is the antisymmetric Levi-Civita symbol. The Landau gauge and Eqs. (3)-(4) can be used for uniform $\mathbf{B}=\mathbf{B}_{0}$ when $\delta \mathbf{B}$-fluctuations are neglected. In the prevailing part of our study we shall apply the general Coulomb gauge of the field $\mathbf{A}(\mathbf{x})$, which does not exclude spatial dependent magnetic fluctuations $\delta \mathbf{B}(\mathbf{x})$.

In nonmagnetic superconductors, where the mean value $\langle\mathbf{M}\rangle=(\mathbf{M}-\delta \mathbf{M})$ of magnetization $\mathbf{M}$ is equal to zero in the normal state in zero external magnetic field, 
the magnetic induction in the presence of the external magnetic field takes the form:

$$
\mathbf{B}=\mathbf{H}_{0}+\delta \mathbf{H}(\mathbf{x})+4 \pi \delta \mathbf{M}(\mathbf{x}),
$$

where $\mathbf{H}_{0}$ is the (uniform) regular part of the external magnetic field and $\delta \mathbf{H}$ is an irregular part of $\mathbf{H}$ created by uncontrollable effects. We neglect the irregular part $\delta \mathbf{H}$ and set $\mathbf{H}_{0}=0$, then $\mathbf{B}$ contains only a fluctuation part $\mathbf{B} \equiv \delta \mathbf{B}(\mathbf{x})=4 \pi \delta \mathbf{M}(\mathbf{x})$ that describes the diamagnetic variations of $\mathbf{M}(\mathbf{x})$ around the zero value $\langle\mathbf{M}\rangle=0$ due to fluctuations $\delta \psi(\mathbf{x})$ of the ordering field $\psi(\mathbf{x})$ above $\left(T>T_{c 0}\right)$ and below $\left(T<T_{c 0}\right)$ the normalto-superconducting transition at $T_{c 0}$. Note that the nonfluctuation part $\mathbf{A}_{0}=[\mathbf{A}(\mathbf{x})-\delta \mathbf{A}(\mathbf{x})]$ corresponds to the regular part $\mathbf{B}_{0}=\left(\mathbf{H}_{0}+\langle\mathbf{M}\rangle\right)=0$ of $\mathbf{B}$ in nonmagnetic superconductors $(\langle\mathbf{M}\rangle=0)$ in zero external magnetic field $\left(\mathbf{H}_{0}=0\right)$. Then we can set $\mathbf{A}_{0}(\mathbf{x})=0$ and, hence, $\delta \mathbf{A}(\mathbf{x})=\mathbf{A}(\mathbf{x})$, so we have an entirely fluctuation vector potential $\mathbf{A}(\mathbf{x})$, which interacts with the order parameter $\psi(\mathbf{x})$. This interaction can be of the type $|\psi|^{2} A$ and $|\psi|^{2} A^{2}$ and generates all the effects discussed in the paper.

We accept periodic boundary conditions for the superconductor surface. This means to ignore the surface energy including the additional energy due to the magnetic field penetration in the surface layer of thickness equal to the London penetration depth $\lambda(T)=\lambda_{0}\left|t_{0}\right|^{-1 / 2}, t_{0}=$ $\left|T-T_{c 0}\right| / T_{c 0} ; \lambda_{0}=\left(m c^{2} b / 8 \pi e^{2} \alpha_{0} T_{c 0}\right)^{1 / 2}$ is the "zerotemperature" value of $\lambda$. This approximation is adequate for superconductors of thickness $L_{0} \gg \lambda(T) \gg a_{0}$, where $a_{0}$ is the lattice constant and $L_{0}=\min \left\{L_{i}, i=\right.$ $1, \ldots, D\}$. As we suppose the external magnetic field to be zero $\left(H_{0}=0\right)$ or very small in real experiments, the requirement $L_{0} \gg \lambda(T)$ can be ignored and we have the simple condition $L_{0} \gg a_{0}$.

In microscopic models of periodic structures the periodic boundary conditions confine the wave vectors $\mathbf{k}_{i}=$ $\left\{k_{i}=\left(2 \pi n_{i} / L_{i}\right) ; i=1, \ldots, D\right\}$ in the first Brillouin zone $\left[-\left(\pi / a_{0}\right) \leq k_{i}<\left(\pi / a_{0}\right)\right]$ and the expansion of their values beyond this zone can be made either by neglecting the periodicity of the crystal structure or on the basis of the assumption that large wave numbers $k=|\mathbf{k}|$ have a negligible contribution to the calculated quantities. The last argument is widely accepted in the phase transition theory, where the long-wavelength limit $\left(k a_{0} \ll 1\right)$ can be used. In particular, this argument is valid in the continuum limit $\left(V_{D} / a_{0}^{D} \rightarrow \infty\right)$. Therefore, for both crystal and nonperiodic structures we can use the cutoff $\Lambda \sim\left(\pi / a_{0}\right)$ and afterwards extend this cutoff to infinity, provided the main contributions in the summations over $\mathbf{k}$ come from the relatively small wavenumbers $(k \ll \Lambda)$. This is in fact a quasimacroscopic description based on the GL functional (1), which means that the microscopic phenomena are excluded from our consideration.

The GL free energy functional takes into account phenomena with characteristic lengths $\xi_{0}$ and $\lambda_{0}$ or larger $(\xi$ and $\lambda)$, where $\lambda(T)$ is the London penetration length mentioned above and $\xi(T)=\xi_{0}|t|^{-1 / 2}$ is the coherence length [2]; here $\xi_{0}=\left(\hbar^{2} / 4 m \alpha_{0} T_{c 0}\right)^{1 / 2}$ is the zero-temperature coherence length. In low-temperature superconductors $\xi_{0}$ and $\lambda_{0}$ are much bigger than the lattice constant $a_{0}$. Having in mind this argument we will assume in our investigation that $\Lambda \ll\left(\pi / a_{0}\right)$. Whether the upper cutoff $\Lambda$ is chosen to be either $\Lambda \sim 1 / \xi_{0}$ or $\Lambda \sim 1 / \lambda_{0}$ is a problem that has to be solved by additional considerations. According to arguments presented in Ref. [30] and Sec. II.F, we will often make the choice $\lambda \sim \xi_{0}^{-1}$.

We will use the Fourier expansion

$$
A_{j}(\mathbf{x})=\frac{1}{V_{D}^{1 / 2}} \sum_{k} A_{j}(\mathbf{k}) e^{i \mathbf{k} \cdot \mathbf{x}}
$$

and

$$
\psi(\mathbf{x})=\frac{1}{V_{D}^{1 / 2}} \sum_{k} \psi(\mathbf{k}) e^{i \mathbf{k} \cdot \mathbf{x}},
$$

where the Fourier amplitudes $A_{j}(\mathbf{k})$ obey the relation $A_{j}^{*}(\mathbf{k})=A_{j}(-\mathbf{k})$ and $\mathbf{k} \cdot \mathbf{A}(\mathbf{k})=0$. The Fourier amplitude $\psi(\mathbf{k})$ is not equal to $\psi^{*}(-\mathbf{k})$ because $\psi(\mathbf{x})$ is complex function. For the same reason $\psi(0) \equiv \psi(\mathbf{k}=0)$ a complex number.

Functional (1) is invariant under global $U(1)$ rotations defined by $\psi(\mathbf{x}) \rightarrow \psi(\mathbf{x}) \exp (i \alpha)$, where the angle $\alpha$ does not depend on the spatial vector $\mathbf{x}$, and under the local $U(1)$ gauge transformations $\psi(\mathbf{x}) \rightarrow \psi(\mathbf{x}) \exp [i \alpha(\mathbf{x})]$, $\mathbf{A}(\mathbf{x}) \rightarrow \mathbf{A}(\mathbf{x})+(\hbar c / 2 e) \nabla \alpha(\mathbf{x})$. According to the discussion in Sec. I, we have to investigate the spontaneous breaking of these symmetries, that is, the ordered phases and the phase transitions in the superconductor. The HLM effect,on which we are going to focus our attention, is one of the results of the local $U(1)$ symmetry breaking.

\section{B. Notes about the MF like approximation}

While the effect of the superconducting fluctuations $\delta \psi(\mathbf{x})$ on the phase transition properties is very weak, as in usual superconductors, and is restricted in a negligibly small vicinity $\left(\left|t_{0}\right| \sim 10^{-12} \div 10^{-16}\right)$ of temperature $T_{c 0}$, we will assume that $\delta \psi(\mathbf{x})=0$, i.e., $\psi \approx\langle\psi(\mathbf{x})\rangle$; from now on we will denote $\langle\psi(\mathbf{x})\rangle$ by $\psi$. So we apply the mean-field approximation with respect to the order parameter $\psi(\mathbf{x})$. Within this approximation we will take into account the $\delta \mathbf{A}(\mathbf{x})$-fluctuations for $\mathbf{B}_{0}=0$, i.e., $\mathbf{A}(\mathbf{x})=\delta \mathbf{A}(\mathbf{x})$. Furthermore, the $\mathbf{A}(\mathbf{x})$-fluctuations can be integrated out from the partition function, defined by:

$$
Z(\psi)=\int \mathcal{D} A e^{-F(\psi, \mathbf{A}) / k_{\mathrm{B}} T},
$$

where the functional integral $\int \mathcal{D} A$ is given by

$$
\int_{-\infty}^{\infty} \prod_{j=1}^{D} \prod_{x \in V_{D}} d A_{j}(\mathbf{x}) \delta[\operatorname{div} \mathbf{A}(\mathbf{x})]
$$

The integration is over all possible configurations of the field $\mathbf{A}(\mathbf{x})$; the $\delta$-function takes into account the Coulomb gauge. 
The partition function $Z(\psi)$ corresponds to an effective free energy $\mathcal{F}_{\mathcal{D}}$ of the $D$-dimensional system:

$$
\mathcal{F}_{D}=-k_{\mathrm{B}} T \ln Z(\psi) .
$$

The magnetic fluctuations will be completely taken into account, if only we are able to solve exactly integral (8). The exact solution can be done for a uniform order parameter $\psi$. The uniform value of $\psi$ is different from the mean-field value of $\psi$, because the uniform fluctuations of $\psi(\mathbf{x})$ always exist, so we should choose one of these two possibilities $[30,35]$. The problem of this choice arises after calculating the integral (8) at the next stage of consideration when effective free energy $\mathcal{F}_{\mathcal{D}}$ is analyzed and the properties of the superconducting phase $(\psi>0)$ are investigated. The effective free energy is a particular case of the effective thermodynamic potential in the phase transition theory $[4,5]$ and we must treat the uniform $\psi$ in the way prescribed in the field theory of phase transitions. It will become obvious from the next discussion that we will use a loop-like expansion, which can be exactly summed up to give a logarithmic dependence on $|\psi|$.

Due to the spontaneous symmetry breaking of the global $U(1)$ continuous symmetry of the ground state $\psi \neq 0$, the effective free energies discussed in this Section depend on the modulus $|\psi|$ of the complex number $\psi=|\psi| e^{i \theta}$ but not on the phase angle $\theta$, which remains arbitrary. That is why we will consider the modulus $|\psi|$ as an "effective order parameter" as the angle $\theta$ does not play any role in the phenomena investigated in this Section. The quantity $|\psi|$ remains undetermined up to the stage when we define the equilibrium order parameter $\left|\psi_{0}\right|$ by the equation of state $\left[\partial \mathcal{F}_{D}(\psi) / \partial \psi\right]=0$. This equation gives the equilibrium value $\psi_{0}$ of $\psi$ and the difference $\delta \psi_{0}=\left(\psi_{0}-\psi\right)$ can be treated as the uniform (zero dimensional) fluctuation of the field $\psi(\mathbf{x})$. The $\mathbf{x}$ dependent fluctuations $\delta \psi(\mathbf{x})$ have been neglected because of the uniformity of $\psi$. The solution $\psi_{0}$ will be stable towards the uniform fluctuation $\delta \psi$, provided the same solution $\psi_{0}=\left|\psi_{0}\right| e^{i \theta_{0}}$ corresponds to a stable (normal or superconducting) phase; the phase angle $\theta_{0}$ remains unspecified. We begin our investigation by setting $\psi$ uniform but at some stage we will also ignore the uniform fluctuation $\delta \psi$ and deal only with the equilibrium value $\psi_{0}$ of $\psi$. The equilibrium value will be calculated after taking into account magnetic fluctuations, so it will be different from the usual result $\left|\psi_{0}\right|=(|a| / b)^{1 / 2}[2]$ where both magnetic and superconducting fluctuations are ignored. This simplest approximation for the equilibrium value of $\psi$ is obtained from the GL free energy (1), provided $e=0$ and the gradient term is neglected. Hereafter we will keep the symbol $\left|\psi_{0}\right|$ for the equilibrium order parameter in the more general case when the magnetic fluctuations are not neglected and will denote the same quantity for $e=0$ by $\eta \equiv\left|\psi_{0}(e=0)\right|=(|a| / b)^{1 / 2}$.

The above described approximation neglects the saddle point solutions of GL equations, where $\langle\psi(\mathbf{x})\rangle$ is $\mathbf{x}$ dependent. Therefore, the vortex state that is stable in type II superconductors cannot be achieved. This is consistent with setting the external magnetic field to ze- ro, so the vortex state cannot occur in any type superconductor. These arguments can be easily verified with the help of GL equations [2] for zero external magnetic field; the only nonzero solution for $\psi$ in this case is given by $\eta=(|a| / b)^{1 / 2}$ although the magnetic fluctuations $\mathbf{A}(\mathbf{x})=\delta \mathbf{A}(\mathbf{x})$ are properly considered.

In conclusion we can argue that the described method will be convenient for both type I and type II superconductors in zero external magnetic field, if the $\psi$ fluctuations have a negligibly small effect on phase transition properties $T_{c 0}=T_{c}\left(H_{0}=0\right)$, where $T_{c}$ denotes the phase transition line for any $H_{0} \geq 0$. For type II superconductors in $H_{0}>0$, two lines $T_{c 1}\left(H_{0}\right)$ and $T_{c 2}\left(H_{0}\right)$ should be defined, usually given by $H_{c 1}(T)$ and $H_{c 2}(T)[2]$.

\section{Effective free energy}

When the order parameter $\psi$ is uniform the functional (1) is reduced to

$$
F(\psi, \mathbf{A})=F_{0}(\psi)+F_{A}(\psi)
$$

with

$$
F_{0}(\psi)=V_{D}\left(a|\psi|^{2}+\frac{b}{2}|\psi|^{4}\right)
$$

and

$$
\begin{aligned}
F_{A}(\psi) & =\frac{1}{8 \pi} \int d^{D} x\left\{\rho(\psi) \mathbf{A}^{2}(\mathbf{x})\right. \\
& \left.+\frac{1}{2} \sum_{i, j=1}^{D}\left(\frac{\partial A_{j}}{\partial x_{i}}-\frac{\partial A_{i}}{\partial x_{j}}\right)^{2}\right\} .
\end{aligned}
$$

Here $\rho=\rho_{0}|\psi|^{2}$ and $\rho_{0}=\left(8 \pi e^{2} / m c^{2}\right)$. It is convenient to calculate the partition function $Z(\psi)$ and the effective free energy $\mathcal{F}_{\mathrm{D}}(\psi)$ in the $\mathbf{k}$-space, where Eqs. (9) and (13) take the form

$$
\int_{-\infty}^{\infty} \prod_{j=1}^{D} \prod_{\mathbf{k}>0}^{k \leq \Lambda} d \operatorname{Re} A_{j}(\mathbf{k}) d \operatorname{Im} A_{j}(\mathbf{k}) \delta[\mathbf{k} \cdot \mathbf{A}(\mathbf{k})]
$$

and

$$
F_{A}(\psi)=F_{A}(0)+\Delta F_{A}(\psi)
$$

Here

$$
F_{A}(0)=\frac{1}{8 \pi} \sum_{j, k} k^{2}\left|A_{j}(\mathbf{k})\right|^{2}
$$

and

$$
\Delta F_{A}(\psi)=\rho \sum_{j, k}\left|A_{j}(\mathbf{k})\right|^{2} ;
$$

note, that we have used the Coulomb gauge $\mathbf{k} \cdot \mathbf{A}(\mathbf{k})=0$.

Then the partition function (8) will be 


$$
\mathcal{Z}(\psi)=e^{-F_{0}(\psi) / k_{\mathrm{B}} T} \mathcal{Z}_{A}(\psi)
$$

where

$$
\mathcal{Z}_{A}(\psi)=\int \mathcal{D} A e^{-F_{A}(\psi) / k_{\mathrm{B}} T}
$$

with $F_{A}(\psi)$ given by (15) and the functional integration defined by the rule (14). With the help of Eqs. (10)-(19) the effective free energy $\mathcal{F}_{D}(\psi)$ becomes

$$
\mathcal{F}_{D}(\psi)=F_{0}(\psi)+\mathcal{F}_{f}(\psi),
$$

where $F_{0}(\psi)$ is given by Eq. (12) and

$$
\mathcal{F}_{f}(\psi)=-k_{\mathrm{B}} T \ln \left[\frac{\mathcal{Z}(\psi)}{\mathcal{Z}(0)}\right]
$$

is the $\psi$-dependent fluctuation part of $\mathcal{F}(\psi)$. In Eq. (20) the $\psi$-independent fluctuation energy $\left\{-k_{\mathrm{B}} T \ln \left[\mathcal{Z}_{A}(0)\right]\right\}$ has been omitted. This energy should be ascribed to the normal state of the superconductor, which by convention is set equal to zero.

Defining the statistical averages as

$$
\langle(\ldots)\rangle=\frac{\int \mathcal{D} \mathcal{A} e^{-F_{A}(0) / k_{\mathrm{B}} T}(\ldots)}{\mathcal{Z}_{A}(0)},
$$

we can write Eq. (21) in the form

$$
\mathcal{F}_{f}(\psi)=-k_{\mathrm{B}} T \ln \left\langle e^{-\Delta F_{A}(\psi) / k_{\mathrm{B}} T}\right\rangle .
$$

Eq. (23) is a good starting point for the perturbation calculation of $\mathcal{F}_{f}(\psi)$. We expand the exponent in Eq. (23) and also take into account the effect of the logarithm on the infinite series. As a result we obtain

$$
\mathcal{F}_{f}(\psi)=\sum_{l=1}^{\infty} \frac{(-1)^{l}}{l !\left(k_{\mathrm{B}} T\right)^{l-1}}\left\langle\Delta F_{A}^{l}(\psi)\right\rangle_{c},
$$

where $\langle\ldots\rangle_{c}$ denotes connected averages [4]. Now we have to calculate averages of the type

$$
\left\langle A_{\alpha}\left(\mathbf{k}_{1}\right), A_{\beta}\left(\mathbf{k}_{2}\right) \ldots A_{\gamma}\left(\mathbf{k}_{n}\right)\right\rangle_{c} .
$$

Here we will use the Wick theorem and the correlation function of the form

$$
G_{i j}^{(A)}\left(\mathbf{k}, \mathbf{k}^{\prime}\right)=\left\langle A_{i}(\mathbf{k}) A_{j}\left(-\mathbf{k}^{\prime}\right)\right\rangle=\delta_{\mathbf{k}, \mathbf{k}^{\prime}} G_{i j}^{A}(k),
$$

where

$$
G_{i j}^{A}(\mathbf{k})=\left\langle A_{i}(\mathbf{k}) A_{j}(-\mathbf{k})\right\rangle=\frac{4 \pi k_{\mathrm{B}} T}{k^{2}}\left(\delta_{i j}-\hat{k_{i}} \hat{k_{j}}\right)
$$

and $\hat{k}_{i}=\left(k_{i} / k\right)$.

The calculation of lowest order terms $(l=1,2,3)$ in Eq. (24) with the help of (25) - (27) is straightforward. The perturbation terms in (24) are shown by diagrams in Fig. 1. The infinite series (24) can be exactly summed up and the result is the following logarithmic function

$$
\mathcal{F}_{f}(\psi)=\frac{(D-1)}{2} k_{\mathrm{B}} T \sum_{k} \ln \left[1+\frac{\rho(\psi)}{k^{2}}\right]
$$

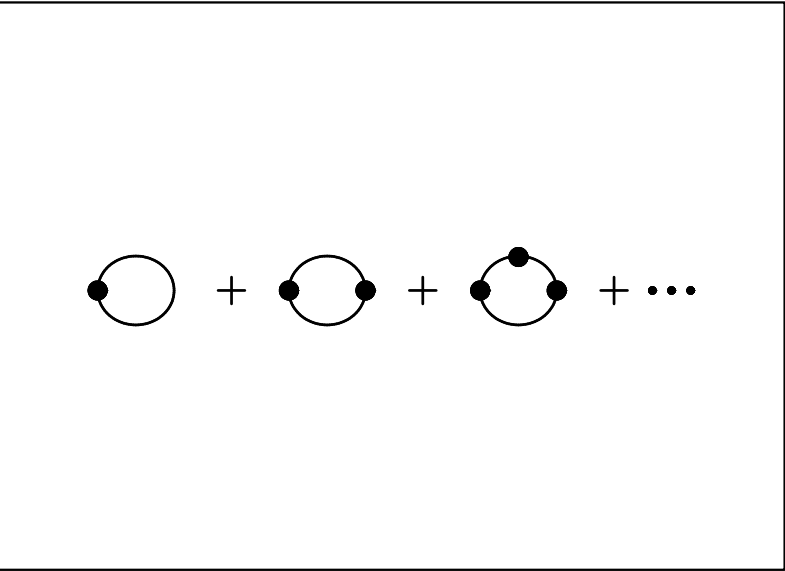

Fig. 1. Diagrammatic representation of the series (24); represents the $\rho$-vertex in (13) and (17), and the solid lines represent bare correlation functions $\left\langle\left|A_{j}(\mathbf{k})\right|^{2}\right\rangle$.

The same result for $\mathcal{F}_{f}(\psi)$ can be obtained by a direct calculation of the Gaussian functional integral (8). This is done using the integral representation of the $\delta$ function in (9) or (14) but it introduces an additional functional integration that should be carried out after the integration over $A_{j}(\mathbf{x})$.

Eqs. (10), (20) and (28) give the effective free energy density

$$
f_{D}(\psi)=\mathcal{F}_{D}(\psi) / V_{D}
$$

in the form

$$
f_{D}(\psi)=f_{0}(\psi)+\Delta f_{D}(\psi),
$$

where

$$
f_{0}(\psi)=a|\psi|^{2}+\frac{b}{2}|\psi|^{4}
$$

and

$$
\Delta f_{D}(\psi)=\frac{(D-1) k_{\mathrm{B}} T}{2 V_{D}} \sum_{k} \ln \left(1+\frac{\rho}{k^{2}}\right) .
$$

Eqs. (20) and (29) - (32) are the basis of our further consideration. We should mention that the fluctuation contribution $\Delta f_{D}(\psi)$ to $f(\psi)$ transforms to convergent integral in the continuum limit

$$
\frac{1}{V_{D}} \sum_{k} \rightarrow \int \frac{d^{D} k}{(2 \pi)^{D}}=K_{D} \int_{0}^{\Lambda} d k . k^{D-1}
$$

where $K_{D}=2^{1-D} \pi^{-D / 2} / \Gamma(D / 2)$ for all spatial dimensionalities $D \geq 2$. But the terms in the expansion of the logarithm in (32) are power-type divergent with the exception of several low-order terms in certain dimensionalities $D$. Therefore, we will work with a finite sum of an infinite series with infinite terms. In our further calculations we will keep the cutoff $\Lambda$ finite for all relevant terms in $\Delta f_{D}(\psi)$. This is a prerequisite for obtaining correct results. 


\section{2D-3D crossover}

The dimensional (2D-3D) crossover has been considered in Refs. [34,39]. Here we follow Ref. [34], where the effective free energy density $f(\psi) \equiv f_{3}(\psi)=\mathcal{F}_{3}(\psi) / V_{3}$ of a thin superconducting film of the thickness $L_{0}$ and volume $V=V_{3}=\left(L_{0} L_{1} L_{2}\right)$ is derived in a more general way, which allows the investigation of the dimensional crossover. Now one should perform integration (33) only with respect to the wave vector components $k_{1}$, and $k_{2}$ corresponding to the large sizes $L_{j} \gg L_{0}(j=1,2)$. The result for the effective free energy density is [34]:

$$
f(\psi)=a|\psi|^{2}+\frac{b}{2}|\psi|^{4}+k_{\mathrm{B}} T J[\rho(\psi)],
$$

where

$$
J(\rho)=\int_{0}^{\Lambda} \frac{d q}{2 \pi} q S(q, \rho)
$$

is given by the sum

$$
S=\frac{1}{L_{0}} \sum_{k_{0}=-\Lambda_{0}}^{+\Lambda_{0}} \ln \left[1+\frac{\rho(\psi)}{q^{2}+k_{0}^{2}}\right]
$$

and $q=|\mathbf{q}|, \mathbf{q}=\left(k_{1}, k_{2}\right)$.

In Eqs. (34)-(36), the integral $J(\rho)$ and the sum $S(q, \rho)$ over the wave vector $\mathbf{k}=\left(\mathbf{q}, k_{0}\right)$ are truncated by the upper cutoffs $\Lambda$ and $\Lambda_{0}$. The finite cutoff $\Lambda$ is introduced for the wave number $q$ and $\Lambda_{0}$ stands for $k_{0}$.

As our study is based on the quasimacroscopic GL approach the second cutoff $\Lambda_{0}$ should be again related to $\xi_{0}$ rather than to the lattice constant $a_{0}$, i. e. $\Lambda_{0} \sim\left(1 / \xi_{0}\right)$, which means that phenomena at distances shorter than $\xi_{0}$ are excluded from our consideration. We will assume that the lowest possible value of $\Lambda_{0}$ is $\left(\pi / \xi_{0}\right)$, as is for $\Lambda$, but we will keep in mind that both $\Lambda_{0}$ and $\Lambda$ can be extended to infinity, provided the main contribution to the integral $J(\rho)$ and the sum $S$ come from the long wavelength limit $\left(q \xi_{0} \ll 1\right)$.

In a close vicinity of the phase transition point $T_{c 0}$ from normal $(\psi=0)$ to Meissner state $(|\psi|>0)$ the parameter $\rho \sim|\psi|^{2}$ is small and the main contribution to the free energy $f(\psi)$ will be given by the terms in $S$ with small wave vectors $k \ll \Lambda$. This allows an approximate but reliable treatment of the $2 \mathrm{D}-3 \mathrm{D}$ crossover by expanding the summation over $k_{0}$ in (36) to infinity, $\Lambda_{0} \sim \infty$. A variant of the theory when $\Lambda_{0}$ is kept finite $\left(\Lambda=\Lambda_{0}=\pi / \xi_{0}\right)$ can also be developed but the results are too complicated [36]. Performing the sum- mation and the integration in Eqs. (35)-(36) we obtain $J(\rho)=\left(\Lambda^{2} / 2 \pi L_{0}\right) I(\rho)$, where

$$
I(\rho)=\int_{0}^{1} d y \ln \left[\frac{\sinh \left(\frac{1}{2} L_{0} \Lambda \sqrt{\rho+y}\right)}{\sinh \left(\frac{1}{2} L_{0} \Lambda \sqrt{y}\right)}\right]
$$

The integral (37) has a logarithmic divergence that corresponds to the infinite contribution of magnetic fluctuations to the free energy of the normal phase $\left(T_{c 0}>\right.$ $0, \varphi=0)$. Such a type of divergence is a common property of many phase transition models. In the present case, as is in other systems, this divergence is irrelevant, because the divergent term does not depend on the order parameter $\psi$ and the free energy $f(\psi)$ is defined as the difference between the total free energies of the superconducting and normal phases: $f(\psi)=\left(f_{\mathrm{S}}-f_{\mathrm{N}}\right)$.

Introducing a dimensionless order parameter $\varphi=$ $\left(\psi / \psi_{0}\right)$, where $\psi_{0}=\left(\alpha_{0} T_{c 0} / b\right)^{1 / 2}$ is the value of $\psi$ at $T=0$, we obtain the free energy (34) in the form

$f(\varphi)=\frac{H_{c 0}^{2}}{8 \pi}\left[2 t_{0} \varphi^{2}+\frac{b}{2}|\varphi|^{4}+2\left(1+t_{0}\right) C I\left(\mu \varphi^{2}\right)\right]$,

with $I\left(\mu \varphi^{2}\right)$ given by Eq. (37), $\mu=(1 / \pi \kappa)^{2}, \Lambda=\pi / \xi_{0}$, and

$$
C=\frac{2 \pi^{2} k_{\mathrm{B}} T_{c 0}}{L_{0} \xi_{0}^{2} H_{c 0}^{2}}
$$

From the equation of state $(\partial f / \partial \varphi=0)$ we find two possible phases: $\varphi_{00}=0$ and the superconducting phase $\left(\varphi_{0}>0\right)$ defined by the equation

$$
t_{0}+\varphi_{0}^{2}+\frac{\left(1+t_{0}\right) C L_{0} \xi_{0}}{4 \pi \lambda_{0}^{2}} K\left(\mu \varphi_{0}^{2}\right)=0
$$

where

$$
K(z)=\int_{0}^{1} d y \frac{\operatorname{coth}\left(\frac{1}{2} L_{0} \Lambda \sqrt{y+z}\right)}{\sqrt{y+z}} .
$$

The analysis of the stability condition $\left(\partial^{2} f / \partial \varphi^{2} \geq 0\right)$ shows that the normal phase is a minimum of $f(\varphi)$ for $t_{0} \geq 0$, whereas the superconducting phase is a minimum of $f(\varphi)$ if

$$
1>\frac{1}{4}\left(1+t_{0}\right) C L_{0} \Lambda \mu^{2} \tilde{K}\left(\mu \varphi_{0}^{2}\right)
$$

where

$$
\tilde{K}(z)=\int_{0}^{1} \frac{d y}{y+z}\left[\frac{\operatorname{coth}\left(\frac{1}{2} L_{0} \Lambda \sqrt{y+z}\right)}{\sqrt{y+z}}+\frac{L_{0} \Lambda}{2 \sinh ^{2}\left(\frac{1}{2} L_{0} \Lambda \sqrt{y+z}\right)}\right] .
$$


The entropy jump $\Delta s=(\Delta S / V)=\left[-d f\left(\varphi_{0}\right) / d T\right]$ per unit volume at the equilibrium point of the phase transition $T_{c} \neq T_{c 0}$ is obtained in the form

$$
\Delta s\left(T_{c}\right)=-\frac{H_{c 0}^{2} \varphi_{c 0}^{2}}{4 \pi T_{c 0}}\left[1+\frac{C I\left(\varphi_{c 0}\right)}{\varphi_{c 0}^{2}}\right]
$$

where $\varphi_{c 0} \equiv \varphi_{0}\left(T_{c}\right)$ is the jump of the dimensionless order parameter at $T_{c}$.

The second term in $\Delta s$ can be neglected. In fact, taking into account the equation $f\left[\varphi_{0}\left(T_{c}\right)\right]=0$ for the equilibrium phase transition point $T_{c}$ we obtain that $\left|C I\left(\varphi_{0}\right) / \varphi_{0}^{2}\right|$ is approximately equal to $\left|t_{c 0}+\varphi_{c 0}^{2} / 2\right|$, where $\varphi_{c 0}^{2}$ and the dimensionless shift of the transition temperature $t_{c 0}=t_{0}\left(T_{c}\right)$ are expected to be much smaller than unity. The latent heat $Q=T_{c} \Delta s\left(T_{c}\right)$ and the jump of the specific heat capacity at $T_{c}, \Delta C=$ $T_{c}(\partial \Delta S / \partial T)$ can be easily calculated with the help of Eq. (44). For this purpose we need the function $\varphi_{0}(T)$, which cannot be obtained analytically from Eq. (40).
Eqs. (38) and (40) can be analyzed numerically. This relatively simple $2 \mathrm{D}-3 \mathrm{D}$ crossover formulae can be used in investigations of specific substances by variation of the thickness $L_{0}$ of the films from $L_{0} \gg \Lambda^{-1}$ (3D system) to $a_{0}<L_{0} \ll \Lambda^{-1}$ (quasi-2D system) and even to a $2 \mathrm{D}$ system for $L_{0}=a_{0}$. Then one can vary the effective dimension of the system $D_{\text {eff }}\left(L_{0} \Lambda\right)$ as a function of $L_{0} \Lambda_{0}$ from $D=2$ to $D=3$ [43]. However, from a purely calculational point of view we have found it more convenient to consider particular dimensions of interest separately and then to compare the results in order to demonstrate the relevant differences between the bulk (3D) and thin film properties. This approach is applied below.

\section{E. Effective free energy for particular dimensions}

For purely $2 \mathrm{D}$ superconductor consisting of a single atomic layer, we can use Eqs. (29)-(32) setting $D=2$ and calculate $\Delta f_{2}(\psi)$ with the help of rule (33):

$$
\Delta f_{2}(\psi)=\left(\frac{k_{\mathrm{B}} T}{8 \pi}\right)\left[\left(\Lambda^{2}+\rho_{0}|\psi|^{2}\right) \ln \left(1+\frac{\rho_{0}|\psi|^{2}}{\Lambda^{2}}\right)-\rho_{0}|\psi|^{2} \ln \left(\frac{\rho_{0}|\psi|^{2}}{\Lambda^{2}}\right)\right] .
$$

The first term of this free energy can be expanded in the powers of $|\psi|^{2}$ :

$$
\Delta f_{2}(\psi)=\left(\frac{k_{\mathrm{B}} T}{8 \pi}\right)\left\{\rho_{0}|\psi|^{2}+\rho_{0}|\psi|^{2} \ln \left(\frac{\Lambda^{2}}{\rho_{0}|\psi|^{2}}\right)+\frac{\rho_{0}^{2}|\psi|^{4}}{2 \Lambda^{2}}\right\}
$$

In this way we obtain the result from Ref. [40]. This case is of special interest because of the logarithmic term in the Landau expansion for $f(\psi)$ but it has no practical application for the lack of ordering in purely $2 \mathrm{D}$ superconductors.

For quasi-2D superconductors we assume that $(2 \pi / \Lambda)>L_{0} \gg a_{0}$, where $L_{0}$ is the thickness of the superconducting film and the more precise choice of the upper cutoff $\Lambda \ll\left(1 / a_{0}\right)$ for the wave numbers $k_{i}$ is a matter of additional investigation [30]. In order to justify this definition of quasi-2D system one can use the 2D-3D crossover description presented in Sec. II.D. The summation over the wave number $k_{0}=\left(2 \pi n_{0} / L_{0}\right)$ in Eq. (36) cannot be substituted with an integration because $L_{0} \ll L_{j}$ and the dimension $L_{0}$ does not obey the conditions, valid for $L_{j},(j=1,2)$ [41-43]. Therefore, for such $3 \mathrm{D}$ system we must sum over $k_{0}$ and integrate over two other components $\left(k_{1}\right.$ and $\left.k_{2}\right)$ of the wave vector $\mathbf{k}$ (see Sec.II.D). This gives an opportunity for a systematic description of the 2D-3D crossover as shown in Sec. II.D.
In the limiting case of very small thickness the $2 \mathrm{D}-3 \mathrm{D}$ crossover theory (Sec. II.D) leads to a result, which is obtained more simply in an alternative way, namely, by ignoring all terms corresponding to $k_{0} \neq 0$ in the sum in Eq. (32). This corresponds to a supposition that the quasi-2D film thickness cannot exceed $2 \pi \Lambda$. Assuming this point of view the real physical size of the quasi-2D film thickness will depend on the choice of the cutoff $\Lambda$. It is certain at this stage that $\Lambda \geq \xi_{0}$, because the (quasi)phenomenological GL theory does not account for phenomena for the size less than $\xi_{0}$. The upper cutoff $\Lambda$ of wave numbers can be defined in a more precise way at next stages of consideration; see Sec. II.F-II.H).

For a quasi-2D film we have the expression:

$$
\Delta f(\psi)=\frac{2}{L_{0}} \Delta f_{2}(\psi),
$$

where $\Delta f_{2}(\psi)$ is given by Eq. (45).

For bulk (3D) superconductor we obtain:

$$
\Delta f_{3}(\psi)=\frac{k_{\mathrm{B}} T}{2 \pi}\left[\frac{\Lambda^{3}}{3} \ln \left(1+\frac{\rho_{0}|\psi|^{2}}{\Lambda^{2}}\right)+\frac{2}{3} \rho_{0}|\psi|^{2} \Lambda-\frac{2}{3} \rho_{0}^{3 / 2}|\psi|^{3} \arctan \left(\frac{\Lambda}{\sqrt{\rho_{0}|\psi|^{2}}}\right)\right]
$$


The Landau expansion in the powers of $|\psi|$ in this form of $f_{3}(\psi)$ confirms the respective results of Refs. [6,7], moreover it correctly gives the term of type $\rho_{0}^{2}|\psi|^{4}$, which has been considered small and neglected in these papers.

For $4 \mathrm{D}$-systems $\Delta f_{D}(\psi)$ becomes

$$
\Delta f_{4}(\psi)=\frac{3 k_{\mathrm{B}} T}{64 \pi^{2}}\left[\Lambda^{2} \rho_{0}|\psi|^{2}+\Lambda^{4} \ln \left(1+\frac{\rho_{0}|\psi|^{2}}{\Lambda^{2}}\right)-\rho_{0}^{2}|\psi|^{4} \ln \left(1+\frac{\Lambda^{2}}{\rho_{0}|\psi|^{2}}\right)\right] .
$$

The above expression for $\Delta f_{4}(\psi)$ can be also expanded in the powers of $|\psi|$ to show that it contains a term of the type $|\psi|^{4} \ln \left(\sqrt{\rho_{0}}|\psi| / \Lambda\right)$, which produces a first order phase transition; this case is considered in the scalar electrodynamics [14], as mentioned in Sec. I. In our further investigation we will focus our attention on $3 \mathrm{D}$ and quasi-2D superconductors.

The free energy density $\Delta f_{D}(\psi)$ can be expanded in the powers of $|\psi|$ but the Landau expansion can be done only in an incomplete way for even spatial dimensions. Thus $f_{2}(\psi), f_{4}(\psi)$, and $f(\psi)$ - the free energy density corresponding to the quasi-2D films - contain logarithmic terms, which should be kept in their original form in the further treatment of the function $\Delta f_{D}(\psi)$ in the Landau expansion. The analysis has been performed [35] in two ways: with and without Landau expansion of $\Delta f_{D}(\psi)$. These variants of the theory are called "exact" theory (ET) and "Landau" theory (LT), respectively [33]. It has been shown [35] that these two ways of investigation give the same results in all cases except for quasi-2D films with relatively small thicknesses $\left(L_{0} \ll \xi_{0}\right)$. It seems important to establish the differences between two variants of the theory because the HLM effect is very small and any incorrectness in the theoretical analysis may be a cause for an incorrect result. By the same arguments one can investigate the effect of the factor $T$ in $\Delta f_{D}(\psi)$ on the thermodynamics of quasi-2D films. This factor can be represented as $T=T_{c 0}\left(1+t_{0}\right)$ and one may expect that the usual approximation $T \approx T_{c 0}$, which is well justified in the Landau theory of phase transitions $[2,4]$, may be applied. This way of approximation can be made by neglecting terms in the thermodynamic quantities smaller than the leading ones. On the other hand, practical calculations lead to the conclusion that this approximation cannot be made without a preliminary examination because for some quasi-2D films it produces a substantial error of about $10 \%$ [35]. LT, in which the factor $T$ is substituted by $T_{c 0}$, is referred to as a "simplified Landau expansion" (SLT). All three variants of the theory, ET, LT and SLT, have been investigated in Ref. [35].

\section{F. Limitations of the theory}

The general result (29)-(32) for the effective free energy $f(\psi)$ has the same domain of validity [2] as the GL free energy functional in the zero external magnetic field. When we neglect a sub-nano interval of temperatures near the phase transition point we can use Eq. (1), provided $\left|t_{0}\right|=\left|T-T_{c 0}\right| / T_{c 0}<1$, or in a particular case of type I superconductors, $\left|t_{0}\right|<\kappa^{2}$ [2]. Note, that the latter inequality does not appear in the general GL approach. It comes as a condition for the consistency of this approach with the microscopic BCS theory for type I superconductors [2].

Taking the continuum limit we have to assume that all dimensions of the body, including the thickness $L_{0}$, are much larger than the characteristic lengths $\xi$ and $\lambda$. The exception of this rule is when we consider thin films. Especially for thin films of type I superconductors, where $\left((2 \pi / \Lambda)>L_{0} \gg a_{0}\right)$, we should have in mind that $\xi(T)>\lambda(T)$, so the inequalities $\xi>\lambda>\xi_{0}>\lambda_{0}$ hold true in the domain of validity of the GL theory $\left|t_{0}\right|<\kappa^{2}<1$. In Ref. [30] a comprehensive choice of the cutoff $\Lambda$ has been made, namely, $\Lambda=\xi_{0}$ (the problem for the choice of the cutoff $\Lambda$ is discussed also in Sec. II.G-II.H). The respective conditions for quasi-2D films of type II superconductors are much weaker and are reduced to the usual requirements: $\kappa>1 / \sqrt{2},\left|t_{0}\right|<1$ and $(2 \pi / \Lambda)>L_{0} \gg a_{0}$.

If we do a Landau expansion of $f_{D}(\psi)$ in the powers of $|\psi|^{2}$ the condition $\rho \ll \Lambda^{2}$ should be satisfied. In order to evaluate this condition we substitute $|\psi|^{2}$ in $\rho=\rho_{0}|\psi|^{2}$ with $\eta^{2}=|a| / b$, which corresponds to $e=0$. As $\lambda^{2}(T)=$ $1 / \rho$, the condition for the validity of the Landau expansion becomes $[\Lambda \lambda(T)]^{2} \gg 1$, i. e., $\left(\Lambda \lambda_{0}\right)^{2} \gg\left|t_{0}\right|$. Choosing the general form of $\Lambda_{\tau}=\left(\pi \tau / \xi_{0}\right)$, where $\tau$ describes the deviation of $\Lambda_{\tau}$ from $\Lambda_{1} \equiv \Lambda=\left(\pi / \xi_{0}\right)$, we obtain $(\pi \tau \kappa)^{2} \gg\left|t_{0}\right| ; \kappa=\left(\lambda_{0} / \xi_{0}\right)$ is the GL parameter.

Thus we can conclude that in type II superconductors, where $\kappa=\left(\lambda_{0} / \xi_{0}\right)>1 / \sqrt{2}$, the condition $\left(\rho / \Lambda^{2}\right) \ll 1$ is satisfied very well for the values of the cutoff in the interval between $\Lambda=\left(\pi / \xi_{0}\right)$ and $\Lambda=\left(\pi / \lambda_{0}\right)$, i.e., for $1<\tau<(1 / \kappa)$. For type I superconductors, where $\kappa<1 / \sqrt{2}$ the cutoff value $\Lambda \sim\left(1 / \xi_{0}\right)$ leads to the BCS condition $\left(\left|t_{0}\right|<\kappa^{2}\right)$ for the validity of the GL approach. Substantially larger cutoffs $\left(\Lambda \gg \pi / \xi_{0}\right)$, for example, $\Lambda \sim\left(1 / \lambda_{0}\right)$ for type I superconductors with $\kappa \ll 1$ lead to a contradiction between this BCS condition and the requirement $\rho \ll \Lambda^{2}$.

In our calculations we often use another parameter $\mu_{\tau}=(1 / \pi \tau \kappa)^{2}$ and, in particular, $\mu \equiv \mu_{1}=(1 / \pi \kappa)^{2}$ and in terms of $\mu$ the condition for the validity of expansion of $f_{D}(\psi)$ becomes $\mu\left|t_{0}\right| \ll 1$, or, more generally, $\mu_{\tau}\left|t_{0}\right| \ll 1$. Choosing $\tau=1 / \pi$ we obtain the BCS criterion for the validity of the GL free energy for type I superconductors [2]. The choice $\tau=\left(\xi_{0} / \pi \lambda_{0}\right)$ corresponds to the cutoff $\Lambda_{\tau}=1 / \lambda_{0}$. As we will see in Sec. II.G the thermodynamics near the phase transition point in $3 \mathrm{D}$ 
systems has no substantial dependence on the value of the cutoff $\Lambda_{\tau}$ but it should be chosen in a way that is consistent with the mean-field-like approximation. The cutoff problem for quasi-2D films has been investigated in Ref. [35]. It has been shown that the choice $\Lambda \sim \pi / \xi_{0}$ is consistent in the framework of the GL theory.

Alternatively, the inequality $\left(\rho / \Lambda^{2}\right) \ll 1$ may be investigated with the help of the reduced order parameter $\varphi$ defined by $\varphi=|\psi| / \eta_{0}$, where $\eta_{0} \equiv \eta(T=0)=$ $\left(\alpha_{0} T_{c 0} / b\right)^{1 / 2}$ is the so-called zero-temperature value of the order parameter for the GL free energy $f_{0}(\psi)$, given by Eq. (31). The reduced order parameter $\varphi$ will be equal to $\left|t_{0}\right|$ for $t_{0}<0$, if only the magnetic fluctuations are ignored, i. e., when $|\psi|=\eta$. Using the notation $\varphi$, we obtain the condition $\left(\rho / \Lambda^{2}\right) \ll 1$ in the form $\mu_{\tau} \varphi^{2} \ll 1$. This condition seems to be more precise because it takes into account the effect of magnetic fluctuations on the order parameter $\psi$.

\section{G. Bulk superconductors}

\section{Thermodynamics}

The effective free energy $f_{3}(\psi)$ of bulk (3D-) superconductors is given by Eqs. (29)-(31) and (48). The analytical treatment of this free energy can be done by the Landau expansion in small $\left(\sqrt{\rho_{0}}|\psi| / \Lambda\right)$. Up to the order of $|\psi|^{6}$ we obtain

$$
f_{3}(\psi) \approx a_{3}|\psi|^{2}+\frac{b_{3}}{2}|\psi|^{4}-q_{3}|\psi|^{3}+\frac{c_{3}}{2}|\psi|^{6},
$$

where

$$
\begin{gathered}
a_{3}=a+\frac{k_{\mathrm{B}} T \Lambda \rho_{0}}{2 \pi^{2}}, \\
b_{3}=b+\frac{k_{\mathrm{B}} T \rho_{0}^{2}}{2 \pi^{2} \Lambda}, \\
q_{3}=\frac{k_{\mathrm{B}} T \rho_{0}^{3 / 2}}{6 \pi},
\end{gathered}
$$

and

$$
c_{3}=-\frac{k_{\mathrm{B}} T \rho_{0}^{3}}{6 \pi^{2} \Lambda^{3}}
$$

The cutoff $\Lambda$ in Eqs. (51)-(54) is not specified and can be written in the form $\Lambda_{\tau}=\left(\pi \tau / \xi_{0}\right)$ as suggested in Sec. II.F.

It can be shown by both analytical and numerical calculations [31] that $|\psi|^{6}$-term has no substantial effect on the thermodynamics described by the free energy (50). That is why we ignore this term. The possible phases $\left|\psi_{0}\right|$ are found as a solution of the equation of state:

$$
[\partial f(\psi) / \partial|\psi|]_{\psi_{0}}=0
$$

There always exists a normal phase $\left|\psi_{0}\right|=0$, which is a minimum of $f_{3}(\psi)$ for $a_{3}>0$. The possible superconducting phases are given by

$$
\left|\psi_{0}\right|_{ \pm}=\frac{3 q_{3}}{4 b_{3}}\left(1 \pm \sqrt{1-\frac{16 a_{3} b_{3}}{9 q_{3}^{2}}}\right) \geq 0 .
$$

Having in mind the existence and stability conditions of the $\left|\psi_{0}\right|_{ \pm}$-phases [4] we obtain that the $\left|\psi_{0}\right|_{+}$-phase exists for $\left(16 a_{3} b_{3}\right) \leq 9 q_{3}^{2}$ and this region of existence always corresponds to a minimum of $f_{3}(\psi)$. The $\left|\psi_{0}\right|$--phase exists for $0<a_{3}<9 q_{3}^{2} / 16 b_{3}$ and this region of existence always corresponds to a maximum of $f_{3}(\psi)$, i. e., this phase is absolutely unstable. For $a_{3}=0,\left|\psi_{0}\right|_{-}=0$ and hence, it coincides with the normal phase. For $9 q_{3}^{2}=16 a_{3} b_{3}$ we have $\left|\psi_{0}\right|_{+}=\left|\psi_{0}\right|_{-}=3 q_{3} / 4 b_{3}$ and $f_{3}\left(\left|\psi_{0}\right|_{+}\right)=$ $f_{3}\left(\left|\psi_{0}\right|_{-}\right)=27 q_{3}^{4} / 512 b_{3}^{3}$. Furthermore, $f_{3}\left(\left|\psi_{0}\right|_{-}\right)>0$ for all allowed values of $\left|\psi_{0}\right|_{-}>0$, whereas $f_{3}\left(\left|\psi_{0}\right|_{+}\right)<0$ for $a_{3}<q_{3}^{2} / 2 b_{3}$, and $f_{3}\left(\left|\psi_{0}\right|_{+}\right)>0$ for $\left(q_{3}^{2} / 2 b_{3}\right)<$ $a_{3}<9 q_{3}^{2} / 16 b_{3}$. The equilibrium temperature $T_{\text {eq }}$ of the first order phase transition is defined by the equation $f\left(\left|\psi_{0}\right|_{+}\right)=0$, which gives the following result:

$$
2 b_{3}\left(T_{\mathrm{eq}}\right) a_{3}\left(T_{\mathrm{eq}}\right)=q_{3}^{2}\left(T_{\mathrm{eq}}\right) .
$$

These results are confirmed by numerical calculations of the effective free energy (50) [31].

The equilibrium entropy jump is $\Delta S=V \Delta s$ and $\Delta s=-\left(d f_{3}(|\psi|) / d T\right)$ can be calculated with the help of Eq. (50) and the equation of state (55):

$$
\Delta s=-\left|\psi_{0}\right|^{2} \Phi\left(\left|\psi_{0}\right|\right),
$$

where $\Phi\left(\left|\psi_{0}\right|\right)$ is the following function:

$$
\Phi(y)=\left(\alpha_{0}+\frac{k_{\mathrm{B}} \Lambda \rho_{0}}{2 \pi^{2}}\right)-\frac{\rho_{0}^{3 / 2} k_{\mathrm{B}}}{6 \pi} y+\left(\frac{k_{\mathrm{B}} \rho_{0}^{2}}{4 \pi^{2} \Lambda}\right) y^{2} .
$$

The specific heat capacity per unit volume $\Delta C=$ $T(\partial \Delta s / \partial T)$ is obtained from (58):

$$
\Delta C=-\left(\frac{T}{T_{c 0}}\right) \frac{\partial\left|\psi_{0}\right|^{2}}{\partial t_{0}} \Phi\left(\left|\psi_{0}\right|\right)
$$

The quantities $\Delta s(T)$ and $\Delta C(T)$ can be evaluated at the equilibrium phase transition point $T_{\text {eq }}$, which is found from Eq. (57):

$\frac{T_{\mathrm{eq}}}{T_{c 0}} \approx 1-\frac{k_{\mathrm{B}} \rho_{0} \Lambda}{2 \pi^{2} \alpha_{0}}+\frac{\left(\rho_{0}^{3 / 2} k_{\mathrm{B}} / 6 \pi\right)^{2}}{b+\left(\rho_{0}^{2} k_{\mathrm{B}} / 2 \pi^{2} \Lambda\right) T_{c 0}}\left(\frac{T_{c 0}}{\alpha_{0}}\right)$,

provided $\left|\Delta T_{c}\right|=\left|T_{c 0}-T_{\text {eq }}\right| \ll T_{c 0}$. Further we will see that condition $\left|\Delta T_{c}\right| \ll T_{c 0}$ is valid in real substances. The second term in r. h. s. of Eq. (61) is a typical negative fluctuation contribution whereas the positive third term in r.h.s. of the same equality is typical of the first-order transitions [4].

To obtain the jumps $\Delta s$ and $\Delta C$ at $T_{\text {eq }}$ we have to put the solution $\left|\psi_{0}\right|+$ found from Eq. (56) in Eqs. (58)-(60). The result will be:

$$
\Delta s=-\frac{q_{3 c}^{2}}{b_{3 c}^{2}}\left\{\alpha_{0}+\frac{k_{\mathrm{B}} \rho_{0} \Lambda}{2 \pi^{2}}-\left(\frac{k_{\mathrm{B}} \rho_{0}^{3 / 2}}{6 \pi}\right)^{2} \frac{T_{\mathrm{eq}}}{b_{3 c}^{2}}\right\},
$$


and

$$
\Delta C=\frac{4 \alpha_{0}}{b_{3 c}}\left(\alpha_{0} T_{c 0}-\frac{q_{3 c}^{2} b}{b_{3 c}^{2}}\right)
$$

where $b_{3 c}$ and $q_{3 c}$ are the parameters $b_{3}$ and $q_{3}$ at $T=T_{\text {eq }}$. As $\left|\Delta T_{c}\right|=\left|T_{c 0}-T_{\text {eq }}\right| \ll T_{c 0}$ we can set $T_{\mathrm{eq}} \approx T_{c 0}$ in r.h.s. of Eqs. (62) and (63) and obtain $q_{3 c} \equiv q_{3}\left(T=T_{\mathrm{eq}}\right) \approx q_{3}\left(T_{c 0}\right)$ and $b_{3 c} \approx b_{3}\left(T_{c 0}\right)$.

The latent heat per unit volume, $Q=-T_{\mathrm{eq}} \Delta s$, of the first order phase transition at $T_{\text {eq }}$ can be calculated from Eq. (62). If we neglect the second and third terms in the r.h.s. of Eq. (62) and the second term in the r.h.s. of Eq. (63) we shall obtain a result for the ratio

$$
(\Delta T)_{\mathrm{eq}}=\frac{Q}{\Delta C},
$$

which is similar to that presented in Ref. [6]. Here we should mention that Eq. (63) gives the jump $\Delta C$ at the equilibrium phase transition point of the first order phase transition, described by $|\psi|^{3}$ term [4], while the specific heat jump considered in Ref. [6] is equal to the specific heat jump at the standard second order transition
$\Delta \tilde{C}=\left(\alpha_{0}^{2} T_{c 0} / b\right)[4]$ and is four times smaller. Therefore, we obtain $(\Delta T)_{\text {eq }}$ four times smaller than the respective value in Ref. [6].

\section{The results for $\mathrm{Al}$}

In order to do the numerical estimates we represent the Landau parameters $\alpha_{0}$ and $b$ with the help of the zero-temperature coherence length $\xi_{0}$ and the zero-temperature critical magnetic field $H_{c 0}$. The connection between them is given by the formulae of the standard GL superconductivity theory [2]: $\xi_{0}^{2}=$ $\left(\hbar^{2} / 4 m \alpha_{0} T_{c 0}\right)$ and $H_{c 0}^{2}=\left(4 \pi \alpha_{0}^{2} T_{c 0}^{2} / b\right)$. The expression for the zero-temperature penetration depth $\lambda_{0}=$ $\left(\hbar c / 2 \sqrt{2} e H_{c 0} \xi_{0}\right)$ is obtained from the above relation and $\lambda_{0}=\left(b / \alpha_{0} T_{c 0} \rho_{0}\right)^{1 / 2}$. We will use the following experimental values of $T_{c 0}, H_{c 0}$ and $\xi_{0}$ for Al given in Table 1. The experimental values for $T_{c 0}, H_{c 0}$ and $\xi_{0}$ vary about $10-15 \%$ depending on the method of measurement and the geometry of the samples (bulk material or films) [44] but such deviations do not affect the results of our numerical investigations.

\begin{tabular}{|c|c|c|c|c|c|}
\hline substance & $T_{c 0}(\mathrm{~K})$ & $H_{c 0}(\mathrm{Oe})$ & $\xi_{0}(\mu \mathrm{m})$ & $\kappa$ & $\left|\psi_{0}\right| \times 10^{-11}$ \\
\hline \hline $\mathrm{W}$ & 0.015 & 1.15 & 37 & 0.001 & 0.69 \\
\hline $\mathrm{Al}$ & 1.19 & 99.00 & 1.16 & 0.010 & 2.55 \\
\hline $\mathrm{In}$ & 3.40 & 281.5 & 0.44 & 0.145 & 2.0 \\
\hline
\end{tabular}

Table 1. Values of $T_{c 0}, H_{c 0}, \xi_{0}, \kappa$, and $\left|\psi_{0}\right|$ for $\mathrm{W}, \mathrm{Al}$, In [44].

The evaluation of the parameters $a_{3}$ and $b_{3}$ for $\mathrm{Al}$ gives:

$$
a_{3}=\left(\alpha_{0} T_{c 0}\right)\left[t_{0}+0.972 \times 10^{-4}\left(1+t_{0}\right) \tau\right],
$$

and

$$
\frac{b_{3}}{b}=1+\frac{0.117}{\tau} .
$$

Setting $\tau=1$ corresponds to the cutoff $\Lambda_{1}=\left(\pi / \xi_{0}\right)$ (Sec II.F). For $\tau=(1 / \kappa)_{A l}=10^{2}$, which corresponds to the much higher cutoff $\Lambda=\left(\pi / \lambda_{0}\right)$, we have $b_{3} \approx b$. i. e., the $\rho_{0}^{2}$-term in $b_{3}$, given by Eq. (52), can be neglected. However, as we can see from Eq. (66), for $\tau=1$ the same $\rho_{0}^{2}$-correction in the parameter $b_{3}$ is of the order $0.1 b$ and cannot be automatically ignored in all calculations, in contrast to the supposition in Refs. [6,7]. However, the more important fluctuation contribution in $3 \mathrm{D}$ superconductors comes from the $\tau$-term in Eq. (65) for the parameter $a_{3}$. This term is of the order $10^{-4}$ for $\tau \sim 1$ and this is consistent with the condition $\left|t_{0}\right|<\kappa^{2} \sim 10^{-4}$ but for $\tau \sim 10^{2}$, i. e., for $\Lambda \sim\left(\pi / \lambda_{0}\right) \sim 10^{6} \mu \mathrm{m}$, the same $\tau$ - term is of the order $10^{2}$, which exceeds the temperature interval $\left(T_{c 0} \pm 10^{-4}\right)$ for the validity of the BCS condition for Al (Sec. II.F).
These results demonstrate that for our theory to be consistent, we must choose the cutoff $\Lambda_{\tau}=\left(\pi \tau / \xi_{0}\right)$, where $\tau$ is not a large number $(\tau \rightarrow 1 \div 10)$. To be more concrete we set $\Lambda=\Lambda_{1}=\left(\pi / \xi_{0}\right)$ as suggested in Ref. [30].

The temperature shift $t_{\text {eq }}=t_{0}\left(T_{\text {eq }}\right)$ for the bulk Al can be estimated with the help of Eq. (61). We obtain that this shift is negative and very small: $t_{\mathrm{eq}} \sim-10^{-4}$. Note that the second term in the r.h.s. of Eq. (61) is of the order $10^{-4}$, provided $\Lambda \sim\left(1 / \xi_{0}\right)$ whereas the third term in the r.h.s. of the same equality is of the order $10^{-5}$. Once again the change of the cutoff $\Lambda$ to values much higher than $\left(\pi / \xi_{0}\right)$ will take the system outside the temperature interval, where the BCS condition for $\mathrm{Al}$ is valid. Let us note that in Ref. [31] the parameter $t$ corresponds to our present notation $t_{0}$. But the numerical calculation of the free energy function $f_{3}(\psi)$ in Ref. [31] was made for the SLT variant of the theory and the shifted parameter $\left(t_{0}+0.972 \times 10^{-4}\right)$ was incorrectly identified with $t$ and this led to the wrong conclusion for its positiveness at the equilibrium phase transition point $T_{\text {eq }}$. As a matter of fact, the shifted parameter $\left(t_{0}+0.972 \times 10^{-4}\right)$ is positive at $T_{\text {eq }}$ but $t_{\text {eq }} \equiv t_{0}\left(T_{\text {eq }}\right)$ is negative, as firstly noted in Ref. [35]. 
Having in mind these remarks when we evaluate $\Delta s$ and $\Delta C$ for bulk Al we can use simplified versions of (62) and (63) which means to consider only the first terms in the r.h.s and to take $q_{3 c} \approx q_{3}$ and $b_{3 c} \approx b$ at $T_{c 0}$. In this way we obtain

$$
Q=-T_{c 0} \Delta s=0.8 \times 10^{-2}\left[\frac{\mathrm{erg}}{\mathrm{K} \cdot \mathrm{cm}^{3}}\right],
$$

and

$$
\Delta C=2.62 \times 10^{3}\left[\frac{\mathrm{erg}}{\mathrm{cm}^{3}}\right] .
$$

The results are consistent with an evaluation of $\Delta C$ for $\mathrm{Al}$ as a jump $\left(\Delta \tilde{C}=\alpha_{0}^{2} T_{c 0} / b\right)$ at the second order superconducting transition point [6] that, as we have mentioned above, is four times smaller than the jump $\Delta C$ given by Eq. (68).

A complete numerical evaluation of the function $f_{3}(\psi)$ and the jump of the order parameter at $T_{\text {eq }}$ for bulk Al was presented for the first time in Ref. [31]. The results there confirm that the order parameter jump and $\mathrm{Q}$ for bulk type I superconductors are very small and can hardly be observed in experiments.

We will finish the presentation of bulk $\mathrm{Al}$ with the discussion of ratio (64). It can be also written in the form

$$
(\Delta T)_{\mathrm{eq}}=\frac{32 \pi}{9}\left(\frac{k_{\mathrm{B}}^{2} T_{c 0}^{2}}{b \alpha_{0}}\right)\left(\frac{e^{2}}{m c^{2}}\right)^{3},
$$

and it differs by a factor $1 / 4$ from the respective result in Ref. [6]. This difference is due to the fact that we take $\Delta C$ as the jump at the first order transition temperature $T_{\text {eq }}$ while in the above cited paper [6] the authors define $\Delta C$ as a hypothetic jump $(\Delta \tilde{C})$ at the standard second order phase transition point. From Eq. (69) we obtain

$$
(\Delta T)_{\mathrm{eq}}=6.7 \times 10^{-12}\left(T_{c}^{3} H_{c 0}^{2} \xi_{0}^{6}\right),
$$

and multiplying the number coefficient in the above expression by 4 we can obtain Eq. (10) presented in Ref. [6].

\section{H. Quasi-2D films}

Following Refs. [32,33] we can present the free energy density $f(\psi)=\left(F(\psi) / L_{1} L_{2}\right)$ in the form

$$
f(\varphi)=\frac{H_{c 0}^{2}}{8 \pi}\left[2 t_{0} \varphi^{2}+\varphi^{4}+C\left(1+t_{0}\right) \Gamma\left(\mu \varphi^{2}\right)\right],
$$

where

$$
\Gamma(y)=(1+y) \ln (1+y)-y \ln y,
$$

To obtain Eqs. (71)-(72) we have set $\Lambda=\left(\pi / \xi_{0}\right)$ and introduced the notation $\varphi=|\psi| / \eta_{0} ; \eta_{0}$ is defined in Sec. II.E. Some of the properties of free energy (71) were analyzed in Ref. [32] for Al films and in Ref. [33] for films of Tungsten (W), Indium (In), and Aluminium (Al). Here we will briefly discuss the main results.
The equilibrium order parameter $\varphi_{0}>0$ corresponding to the Meissner phase can be easily obtained from the equation $\partial f(\varphi) / \partial \varphi=0$ and Eq. (71):

$$
t_{0}+\varphi_{0}^{2}+\frac{C \mu\left(1+t_{0}\right)}{2} \ln \left(1+\frac{1}{\mu \varphi_{0}^{2}}\right)=0 .
$$

The logarithmic divergence in Eq. (73) has no chance to occur because $\varphi_{0}$ is always positive and does not tend to zero.

The largest terms in the entropy jump $\delta s$ and the specific heat jump $\delta C$ at the equilibrium first order phase transition point $T_{\text {eq }}$ are given by $[32,33]$

$$
\delta s=-\frac{H_{c 0}^{2}}{4 \pi T_{c 0}} \varphi_{\mathrm{eq}}^{2},
$$

where $\varphi_{\mathrm{eq}}=\varphi_{0}\left(T=T_{\mathrm{eq}}\right)$, and

$$
\delta C=\frac{H_{c 0}^{2}}{4 \pi T_{c 0}} .
$$

The latent heat of the phase transition [4] is given by $Q=-T_{\text {eq }} \delta s$ and Eqs. (73)-(74). Since the temperatures $T_{\text {eq }}$ and $T_{c 0}$ have very close values, the difference between the values of $Q, \delta s$, and $\delta C$ at $T_{c 0}$ and $T_{\text {eq }}$, respectively, can also be ignored, for example, $\left|\delta C\left(T_{\text {eq }}\right)-\delta C\left(T_{c 0}\right)\right| / \delta C\left(T_{c 0}\right) \ll 1$ and we can use either $\delta C\left(T_{c 0}\right)$ or $\delta C\left(T_{\mathrm{eq}}\right)[32]$.

The equations $(71)(73)$ corresponding to quasi-2D films are quite different from the respective equations for bulk (3D-) superconductors but it is easily seen that the relatively large values of the order parameter jump $\varphi^{2}$ in thin films again correspond to relatively small values of the GL parameter $\kappa$. That is why we consider element superconductors with small values of $\kappa$ and study the effect of this parameter, the critical magnetic field $H_{c 0}$ and the film thickness $L_{0}$ on the properties of the fluctuation-induced first order phase transition.

We use experimental data for $T_{c 0}, H_{c 0}, \xi_{0}$ and $\kappa$ for W, Al, and In published in Ref. [44] (see Table 1). In some cases the GL parameter $\kappa$ can be calculated with the help of the relation $\kappa=\left(\lambda_{0} / \xi_{0}\right)$ and the available data for $\xi_{0}$ and $\lambda_{0}$. In other cases it is more convenient to use the following representation of the zero-temperature penetration depth:

$$
\lambda_{0}=\frac{\hbar c}{2 \sqrt{2} e H_{c 0} \xi_{0}} .
$$

The value of $\left|\psi_{0}\right|$ in Table 1 is found from

$$
\left|\psi_{0}\right|=\left(\frac{m}{\pi \hbar^{2}}\right)^{1 / 2} \xi_{0} H_{c 0} .
$$

The order parameter dependence on the reduced temperature difference $t_{0}$ is shown in Fig. 2 for Al films of different thicknesses. It is readily seen that the behavior of the function $\varphi_{0}\left(t_{0}\right)$ corresponds to a well established phase transition of the first order. The vertical dashed lines in Fig. 2 indicate the respective values of $t_{\text {eq }}=t_{0}\left(T_{\text {eq }}\right)$, at which the equilibrium phase transition occurs as well as the equilibrium jump $\varphi_{0}\left(T_{\text {eq }}\right)=\varphi_{\text {eq }}$ for different thicknesses of the film. The parts of the 
$\varphi_{0}\left(t_{0}\right)$-curves which extend up to $t_{0}>t_{\text {eq }}$ describe the metastable (overheated) Meissner states, which can appear under certain experimental circumstances (see in Fig. 2 the parts of the curves on the r.h.s. of the dashed lines). The value of $\varphi_{\text {eq }}$ and the metastable region de- crease with the increase of the film thickness, which shows that the first order of the phase transition is better pronounced in thinner films and this confirms the conclusion in Ref. [32].

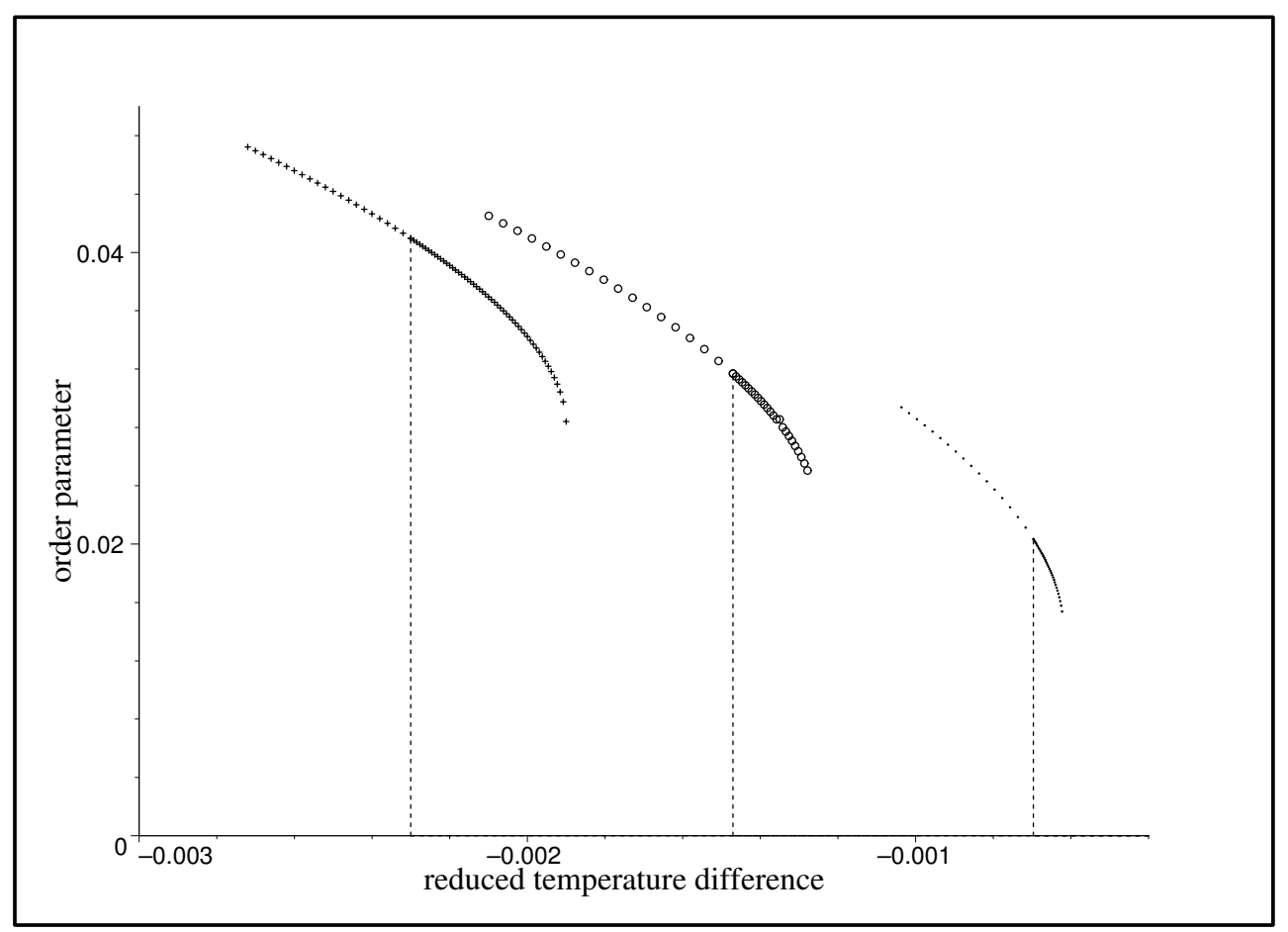

Fig. 2. Order parameter profile $\varphi\left(t_{0}\right)$ of Al films of different thicknesses: $L_{0}=0.05 \mu \mathrm{m}$ ("+"-line), $L_{0}=0.1 \mu \mathrm{m}(\circ)$, and $L_{0}=0.3 \mu \mathrm{m}(\cdot)[33]$.

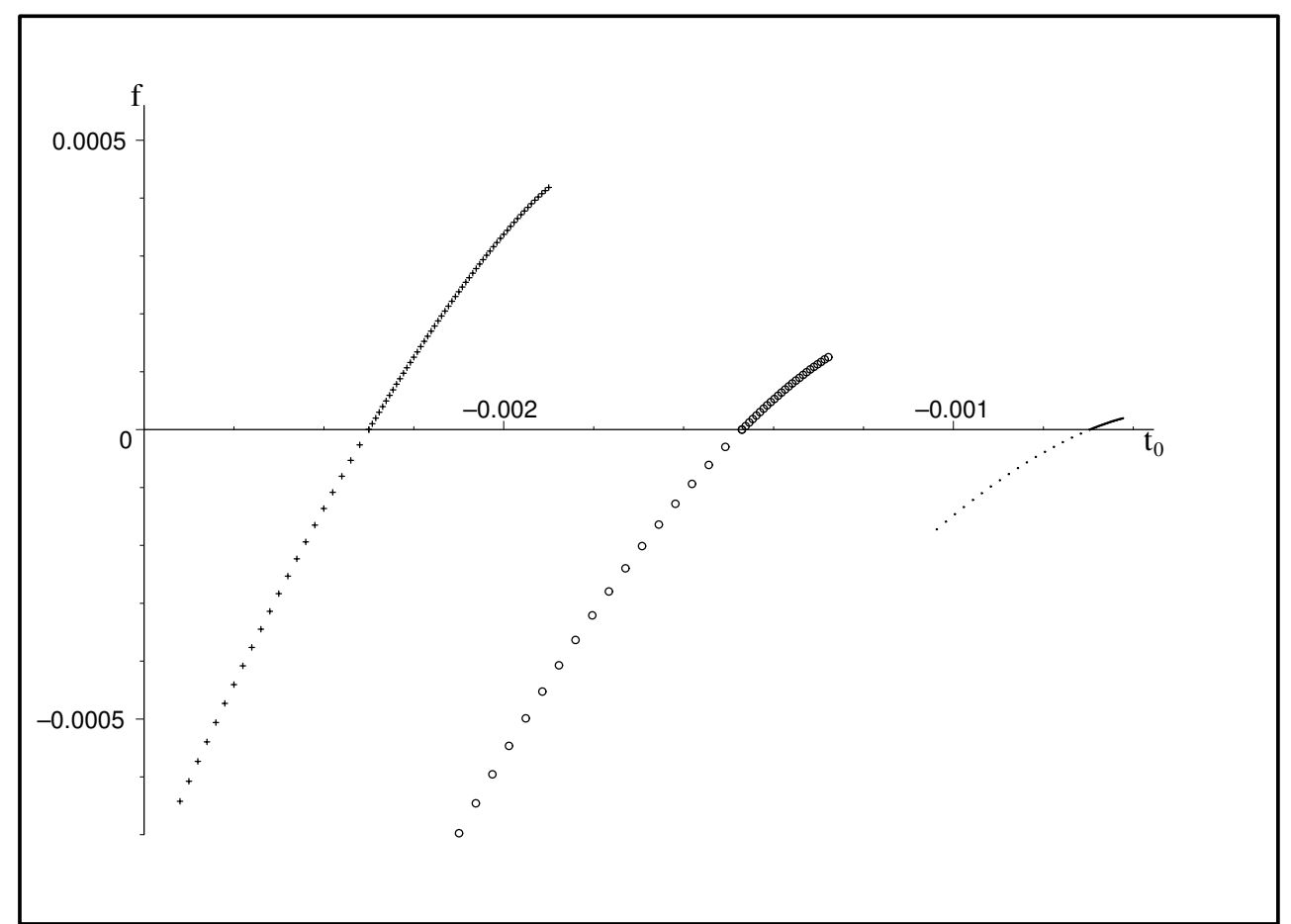

Fig. 3. The free energy $f\left(t_{0}\right)$ for $\mathrm{Al}$ films of the thickness $L_{0}=0.05 \mu \mathrm{m}$ ("+"-line), $L_{0}=0.1 \mu \mathrm{m}(\circ), L_{0}=0.3 \mu \mathrm{m}(\cdot)$ [33]. 


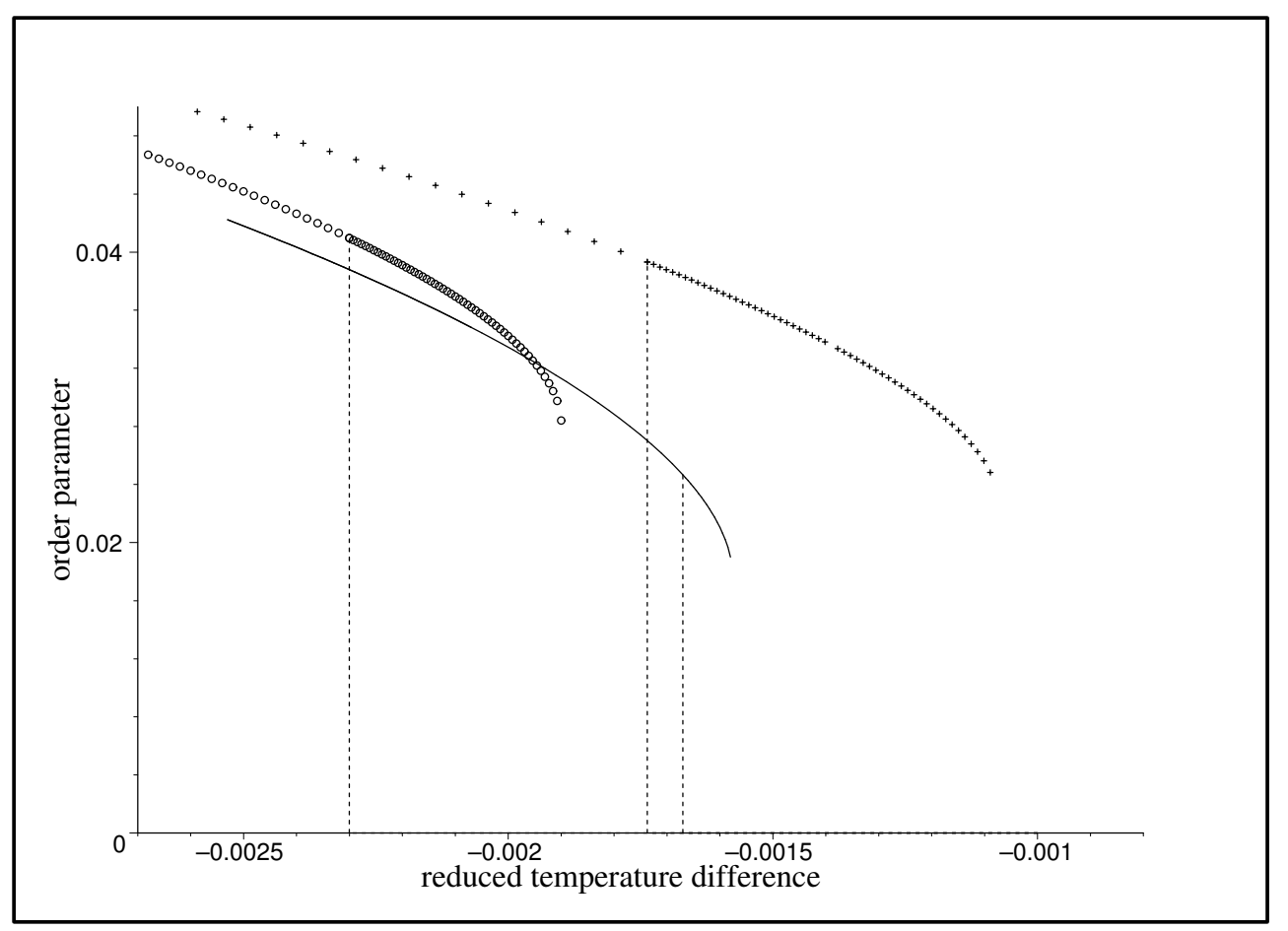

Fig. 4. Order parameter profile $\varphi\left(t_{0}\right)$ films of the thickness $L_{0}=0.05 \mu \mathrm{m}: \mathrm{W}("+$ "-line), $\mathrm{Al}(\circ)$, and In (.) [33].

These results are confirmed by the behavior of the free energy as a function of $t_{0}$. We used Eqs. (71)-(73) for the calculation of the equilibrium free energy $f\left[\varphi_{0}\left(t_{0}\right)\right]$. The free energy for $\mathrm{Al}$ films with different thicknesses is shown in Fig. 3. The equilibrium points $T_{\text {eq }}$ of the phase transition correspond to the intersection of the $f\left(\varphi_{0}\right)$-curves with the $t_{0}$-axis. It is obvious from Fig. 3 that the temperature domain of overheated Meissner states decreases with the increase of the thickness $L_{0}$.

The shape of the equilibrium order parameter $\varphi_{0}\left(t_{0}\right)$ in a broad vicinity of the equilibrium phase transition for thin films $\left(L_{0}=0.05 \mu \mathrm{m}\right)$ of $\mathrm{W}, \mathrm{Al}$, and In was found from Eq. (73). The result is shown in Fig. 4. The vertical dashed lines in Fig. 4 again indicate the respective values of $t_{\mathrm{eq}}=t_{0}\left(T_{\mathrm{eq}}\right)$, at which the equilibrium phase transition occurs as well as the equilibrium jump $\varphi_{0}\left(T_{\text {eq }}\right)=\varphi_{\text {eq }}$ in the different superconductors.

The order parameter jump at the phase transition point of In (the In curve is marked by points in Fig. 4) is relatively smaller than for $\mathrm{W}$, and $\mathrm{Al}$, where the GL parameter has much lower values. The same is valid for the metastability domains; see the parts of the curves in Fig. 4 on the left of the vertical dashed lines. It is obvious from Fig. 4 and Table 2 that the equilibrium jump of the reduced order parameter $\varphi_{\text {eq }}$ of $\mathrm{W}$ has a slightly smaller value than that of $\mathrm{Al}$ although the GL number $\kappa$ for W has a ten times lower value compared with $\kappa$ of Al. Note, that in Fig. 4 we show the jump of $\varphi_{\text {eq }}$, but the important quantity is $|\psi|_{\text {eq }}=\left|\psi_{0}\right| \varphi_{\text {eq }}$. Using the data for $L_{0}=0.05 \mu \mathrm{m}$ from Tables 1 and 2 we find for $|\psi|_{\text {eq }}$ the following values: $0.1 \times 10^{11}$ for $\mathrm{Al}, 0.05 \times 10^{11}$ for In, and $0.02 \times 10^{11}$ for $\mathrm{W}$. This result shows that the value of the critical filed $H_{c 0}$ is also important and should be taken into account together with the smallness of GL number when the maximal values of the order parameter jump are looked for. Thus the value of the order parameter jump at the fluctuation-induced phase transition is maximal, provided small values of the GL parameter $\kappa$ are combined with relatively large values of the critical field $H_{c 0}$. In our case $\mathrm{Al}$ has the optimal values of these two parameters.

The importance of the zero-temperature critical magnetic field $H_{c 0}$ for the enhancement of the jumps of certain thermodynamic quantities at the equilibrium phase transition point $T_{\text {eq }}$ becomes obvious from Eqs. (74), (75) and (77). Eq. (77) shows that the order parameter jump $|\psi|_{\text {eq }}=\left|\psi_{0}\right| \varphi_{\text {eq }}$ is large for large values of $H_{c 0}$ and $\xi_{0}$. This is consistent with the requirement for relatively small values of the GL parameter $\kappa$ as shown by Eq. (76). Therefore, the unmeasurable ratio $Q / \delta C$ discussed in Ref. [6] does not depend on the value of the critical field $H_{c 0}$ but the quantities $Q$ and $\delta C$ themselves as well as the order parameter jump $|\psi|_{\text {eq }}$ depend essentially on $H_{c 0}$. The values of the reduced order parameter jump $\varphi_{\text {eq }}$ for films of $\mathrm{Al}$, In and $\mathrm{W}$ of the same thickness have the same order of magnitude while the respective order parameter jump $|\psi|_{\text {eq }}$ is one order of magnitude higher for $\mathrm{Al}$ than for $\mathrm{W}$, as shown above. The effect of the critical magnetic field $H_{c 0}$ on the latent heat $Q$ is, however, much stronger and, as is seen from Table 2 , the latent heat $Q$ in $\mathrm{W}$ films is very small and can be neglected while in $\mathrm{Al}$ and In films it reaches values, which could be measured in suitable experiments. This is so because the latent heat is proportional to the dif- 
ference $\left[H_{c 0}^{2} / 8 \pi \sim b\left|\psi_{0}\right|^{4}\right]$ between the energies of the ground state (superconducting phase at $T=0$ ) and the normal state. It is consistent with the fact that the fluctuation contribution to the free energy, i. e., the $C$-term in the r. h.s. of Eq. (71) is generated by the term of type $|\psi|^{2} \int d^{D} x \mathbf{A}^{2}(\mathbf{x})$ in the GL free energy. At $T=0$ this free energy term is also proportional to the mentioned difference between the free energies of the ground and normal states.

The shift of the phase transition temperature $t_{\mathrm{eq}}=$ $\left|\left(T_{\text {eq }}-T_{c 0}\right)\right| / T_{c 0}$, the reduced value $\varphi_{\text {eq }}$ of the equilibri- um order parameter jump $|\psi|_{\text {eq }}$, and the latent heat $Q$ of the equilibrium transition are given for films of different thicknesses and substances in Table 2. This table shows that the shift of the phase transition temperature is very small and can be neglected in all calculations and experiments based on them. The values for $\varphi_{\mathrm{eq}}$ for different $L_{0}$ and those for $\left|\psi_{0}\right|$ given in Table 1 confirm the conclusion, which we have made for films of $\mathrm{Al}, \mathrm{In}$, and $\mathrm{W}$ with $L_{0}=0.05 \mu \mathrm{m}$. The latent heat $Q$ has maximal values for In, where the critical field is the highest for the considered materials.

\begin{tabular}{|l|c|c|c|c|c|c|c|c|c|}
\hline \multirow{2}{*}{$L_{0}$} & \multicolumn{3}{|c|}{$\mathrm{Al}$} & \multicolumn{2}{c|}{$\mathrm{In}$} & \multicolumn{3}{c|}{$\mathrm{W}$} \\
\cline { 2 - 10 } & $\mathrm{t}_{\mathrm{eq}}$ & $\varphi_{\mathrm{eq}}$ & $Q$ & $\mathrm{t}_{\mathrm{eq}}$ & $\varphi_{\mathrm{eq}}$ & $Q$ & $\mathrm{t}_{\mathrm{eq}}$ & $\varphi_{\mathrm{eq}}$ & $Q$ \\
\hline \hline 0.05 & -0.00230 & 0.041 & 1.95 & -0.00167 & 0.025 & 3.94 & -0.00174 & 0.039 & $1.6 \times 10^{-4}$ \\
\hline 0.1 & -0.00147 & 0.032 & 0.80 & -0.00094 & 0.017 & 1.82 & -0.00118 & 0.032 & $1.1 \times 10^{-4}$ \\
\hline 0.3 & -0.00070 & 0.023 & 0.41 & -0.00037 & 0.010 & 0.63 & -0.00064 & 0.023 & $5.6 \times 10^{-5}$ \\
\hline 0.5 & -0.00048 & 0.016 & 0.20 & -0.00029 & 0.008 & 0.40 & -0.00048 & 0.020 & $4.1 \times 10^{-5}$ \\
\hline 1 & -0.00029 & 0.012 & 0.11 & -0.00013 & 0.006 & 0.23 & -0.00032 & 0.016 & $2.7 \times 10^{-5}$ \\
\hline 2 & -0.00017 & 0.009 & 0.06 & -0.00008 & 0.004 & 0.10 & -0.00021 & 0.013 & $1.8 \times 10^{-5}$ \\
\hline
\end{tabular}

Table 2. Values of $t_{\mathrm{eq}}, \varphi_{\mathrm{eq}}$, and $Q\left(\mathrm{erg} / \mathrm{cm}^{3}\right)$ for the films of $\mathrm{W}, \mathrm{Al}$, and In with different thicknesses $L_{0}(\mu \mathrm{m})$ [33].

\section{Final remarks}

Our analysis shows that the MF studies of the HLM effect have a well defined domain of validity for both 3Dand quasi-2D superconductors. Our conclusion is that the MF theory of the magnetic fluctuations in superconductors and, in particular, the MF prediction for the fluctuation driven weakly first order phase transition in zero external magnetic field in bulk superconductors [6] and quasi-2D superconducting films $[30,32,33]$ is reliable and can be tested by experiments. While the HLM effect in bulk systems is unobservingly small, in quasi-two dimensional superconductors this effect is much stronger and might be observed with available experimental techniques.

Our consideration of quasi-2D superconductors is highly nontrivial in view of the relevance of the dependence of the effective Landau parameters on the thickness of the films, $L_{0}$. We have justified this dependence by simple heuristic arguments and by a reliable consideration of the 2D-3D crossover. In contrast to initial expectations [6] those films made of superconductors with extremely small GL parameter $\kappa$ such as $\mathrm{Al}$ and, in particular, $\mathrm{W}$ will be the best candidates for an experimental search of the HLM effect; our careful analysis (for the first time published in Ref. [33]) definitely gives a somewhat different answer. The Al films still remain a good candidate for transport experiments, through which the jump of the order parameter at the phase transition point could be measured but surprisingly the $\mathrm{W}$ films turn out inconvenient for the same purpose due to their very low critical field $H_{c 0}$. The importance of the critical magnet- ic field $H_{c 0}$ for the clearly manifested first-order phase transition has been established and discussed, too. Although In has ten times higher GL number $\kappa$ than $\mathrm{Al}$, the In films can be used on equal footing with the $\mathrm{Al}$ films in experiments intended to prove the order parameter jump. Here the choice of one of these materials may depend on other features of experimental convenience. As far as caloric experiments are concerned, the In films seem to be the best candidate due to their high latent heat.

As shown in this review, experiments intended to search the HLM effect can be performed by both type I and type II superconductors. In experiments the external magnetic field cannot be completely eliminated. Then vortex states may occur for $H=|\mathbf{H}|>0$ below $T_{c}=T_{c}(H) \leq T_{c 0}$ in type II superconducting films and this will obscure the HLM effect. Note that both in type I and type II superconductors the external magnetic field $H$ generates additional entropy jump at the phase transition point $T_{c}(H)$ and this effect can hardly be separated from the entropy jump (74) caused by the magnetic fluctuations in the close vicinity of $T_{c 0}$. Therefore, in experiments aimed at the search for the HLM effect the external magnetic field should be minimized as much as possible. For quasi-2D superconductors, where the HLM effect is relatively strong and the latent heat can exceed several ergs, one should ensure external fields less than 10 Oe, or, in more reliable experiments, less than 1 Oe [35].

The temperature range around $T_{c 0}$, where the HLM effect is significant has been estimated in Ref. [6] to a few microdegrees, and later, this estimate has been confirmed in a more accurate way [45]. According to our point of 
view, the possibilities for the observation of the effect are related mainly with its magnitude, because the range of temperatures where it occurs always exists, even in the critical region of strong $\psi$-fluctuations, as the RG studies indicate (Sec. III). The review of the results for $\mathrm{Al}$, In, and W in Sec. II shows that the metastability domains (of overheating and overcooling) are much larger than the Ginzburg critical region. This result confirms the reliability of the MF treatment. As one may see from Figs. 2-4 the metastability temperature interval is relatively larger for smaller values of the GL number $\kappa$, but in order to ensure large values of some measurable thermodynamic quantities as, for example, the latent heat $Q$ and the specific heat capacity $C$ we must choose a material with a large critical field $H_{c}$. The experimental verification of the order parameter jump can be made by transport experiments. As we see from Figs. 2-4 and Table 2, this quantity has maximal values for $\mathrm{W}$, where the parameter $\kappa$ is very small. Having in mind also the large metastability regions in this material one may conclude that $\mathrm{W}$ is a good candidate for a testing the HLM effect by transport experiments (measurements of the superconducting currents), provided some specific disadvantages of this material (quite low $T_{c 0}$, etc.) from the experimental point of view do not contradict to this conclusion. Another effect, which is relevant to the present discussion, is the known variation of the GL parameter $\kappa$ with the variation of the thickness $L_{0}$ (see, e.g., Ref. [46]. The parameter $\kappa<1 / \sqrt{2}$ of a type-I bulk superconductor may change up to the values corresponding to a type-II superconductor with the decrease of the thickness $L_{0}$ below $10^{-7} \mathrm{~m}$.

In Ref. [47] a very interesting interrelationship between the first order phase transition and the vortex fluctuations in type-I 2D superconductors has been analyzed by both analytical and numerical methods. A recent investigation [48] of HLM effect in superconducting films has been proven wrong in our comment [49]; see, also, the Corrigendum [50] to the work [48]. In further papers the same authors and co-workers [51,52] confirm our results published in Refs. [30-35] and discussed in this Section. Another paper has been intended to the treatment of gauge effects in superconductors with the help of the Gaussian effective functional [53], which gives results quite near to the mean-field ones and is useless within the present discussion.

\section{RG STUDIES AND OTHER TOPICS}

\section{A. Notes about RG}

The RG methods are intended to the investigation of the strong fluctuation interactions in the critical region of the second order phase transitions and multi-critical phenomena $[4,5]$. In studies of complex systems with several orderings or with the influence of additional gauge fields, as in Eq. (1), usually the k-space RG methods are used because of their wider applicability. The methods differ from each other in specific technical details but all of them lead to the same predictions about the phase transition properties. The common feature is that the mentioned methods are based on the so-called loop expansion $[4,5,12]$. The RG equations are, in fact, infinite asymptotic series, which are truncated at one-, two-, or, in rare cases, higher orders in the loop expansion. The total infinite series can be summed only in case of trivial (usually exactly soluble) models and in some very exceptional cases as, for example, the RG series for interacting real bosons in the quantum limit $T \rightarrow 0$ [54].

For the simple $\phi^{4}$-theory the RG series can be derived and analyzed to high orders in the loop expansion whereas for more complex models this can be practically done within the one- and two-loop approximations. As the RG series are asymptotic, normally, the one- and two-loop orders give all important features of the specific system of interest: (i) the presence of stable fixed points (FP) of the RG equations and the associated with them types of critical behavior, and (ii) the lack of stable FPs and related conclusions about the lack of standard critical or multi-critical behavior. The higher orders of the theory are more relevant to investigations of the asymptotic properties of the loop series than for obtaining a qualitatively new critical behavior or other new qualitative characteristics of the system. The latter are reliably obtained within the one- and two-loop approximations.

\section{B. The order of the phase transition}

In Ref. [6] the simultaneous effect of $\psi$ - and Afluctuations in the fluctuation Hamiltonian (1) has been investigated by one-loop RG and $\epsilon=(4-d)$-expansion [4] for the general case of $n / 2$-component complex vector field $\psi=\left\{\psi_{\alpha} ; \alpha=1, \ldots, n / 2\right\}$; note that this field is equivalent to a $n$-component real vector field. It has been shown [6] that a stable FP exists below four dimensions $d=(4-\epsilon)$ only for symmetry indices $n \geq 365.9$, which are far above the real symmetry index $n=2$ for usual superconductors and numbers $n=4,6$ corresponding to superconductors with certain unconventional Cooper pairings [55]. For $n<365.9$ real FP does not exist at all. Besides, the $1 / n$-expansion has been used for the calculation [6] of the critical exponents in the so-called large- $n$ limit (alias "Hartree limit") [4,5], where $(n>365.9)$ the phase transition is definitely of the second order.

According to the usual interpretation of RG results, the lack of stable FP is an indication for a lack of standard second order phase transition. But usually additional (non-RG) arguments are needed to determine the actual order of the phase transition. In the present case, the lack of stable FP could be a result of the same mechanism that produces a fluctuation-driven weakly first order phase transition in MF approximation (Sec. II). As RG takes into account both fluctuations of $\psi$ and $\mathbf{A}$, one can conclude that the result for the weakly-first order phase transition in zero external magnetic field is valid for both type I and II superconductors. Of course, the size of the effect (the size of jumps of energy, latent heat, order parameter) will depend on the specific substance. This path of investigations has been further developed 


\section{V. SHOPOVA, T. E. TSVETKOV, D. I. UZUNOV}

in paper [7] and several problems opened in the short Ref. [6], have been solved.

In Ref. [40] where both 2D and 3D superconductors are considered the HLM effect has been confirmed in one-loop order (annealed disorder [40]) just as the availability of the second order phase transition for largen has been proven in another variant of the Hartree limit $(n \rightarrow \infty)$.

Next, the effect has been confirmed in a RG investigation of the equation of state below the phase transition point $T_{c 0}[56,57]$. These investigations show that the effect may occur under quite restricted conditions for the vertex parameters $e$ and $b$ of functional (1), in particular, the effective charge $\left|e^{*}\right|$ exceeds some value. A strong restriction on the HLM effect, but in the opposite direction - a requirement for a sufficiently small effective electric charge $\left|e^{*}\right|$, has been found also by a Monte Carlo (MC) study [58] of a lattice version of model (1). Another MC study [59] concludes that at fixed effective charge $\left(\left|e^{2}\right|=5\right)$ the HLM effect strongly depends on the GL parameter $\kappa=\lambda / \xi$ : it is well established for $\kappa \ll 1$, becomes very weak for $\kappa \sim 1$ and vanishes for large $\kappa$. On the basis of this picture a proposal is made [59] for the existence of a tricritical point at some "tricritical" value of $\kappa$. As $\kappa$ is related with the charge $e$, this proposal seems to be in conformity with other investigations. Note, that the first prediction for change of the phase transition order with the variation of $\kappa$ and for the possibility of "tricritical point" to appear at some value of $\kappa$, has been made in Ref. [60] on the basis of duality arguments [61] and analytical calculations; see further development of this approach in Refs. [62-65]. A further MC study [66] of type II superconductors indicates that these systems undergo a second order phase transition of universality class 3D $X Y$ rather than a weakly first order phase transition. The two-loop RG studies [67,68] show that the account of higher orders of the loop expansion allows a lowering of the critical value $n_{c}=365.9$ but the $\mathrm{RG}$ equations still have no real $\mathrm{FP}$ at $D=3$ and $n=2$ (conventional 3D superconductors), except for a treatment by Padè analysis [68] (see, also, the review [37]). In $2+\epsilon$ dimensions, the nonlinear $\sigma$ model exhibits a second-order phase transition for all values of $n>0$ [69]. Owing to this result one may suppose that the critical value $n_{c}$ vanishes at some dimension $2<d<4$, as mentioned in Ref. [70]. RG studies [71,72] at fixed dimension $d$ have also been carried out with the aim to determine the phase transition order (see, also Ref. [70] for a comment).

\section{Disorder effects}

Here we will discuss mainly disorder described by Gaussian distributions [4]. The effects of annealed and quenched disorder in classical versions of Abelian-Higgs models, equivalent to the GL model (1) have been investigated by Hertz $[73,74]$ in the context of the theory of spin glasses in case of Dzyaloshinskii-Moriya interaction. The specific feature of this approach is that the disorder is associated with the vector gauge field $\mathbf{A}(\mathbf{x})$ and can be used to describe superconductors in random, uncorrelated, or, which is the same, short-range $(\delta-)$ correlated magnetic fields rather than usual ones. In high energy physics this approach makes contact with gauge fields where the Higgs field is coupled to a random color field. It is natural to expect, as has been proven [40,73], that the annealed gauge model will bring a fluctuation-driven first order phase transition at usual symmetry indices and a second order phase transition in the Hartree limit $(n \rightarrow \infty)$. In Ref. [40] the phase transition in case of quenched impurities has been predicted to be of second order in a calculation to one-loop order, in contradiction to the conclusion for a lack of stable nontrivial FP of the RG equations within the same one-loop order [73]. The origin of this discrepancy is clear from the argument that the lack of stable FP may result without any change of the Hamiltonian structure, whereas the conclusion for the second order of the transition in Ref. [40] has been made, perhaps, incorrectly, based on the argument of the absence of $|\psi|^{4}$-term for $D=3$ and of the absence of $|\psi|^{2} \ln |\psi|$-term for $D=2$.

A more usual case of quenched disorder in AbelianHiggs models has been considered in Refs. [75, 76]. This is the case when the parameter $a$ in (1) has a random part, which obeys a Gaussian distribution. This disorder is of the type of random impurities, or, what is the same, "random critical temperature [4]. In the case of the so-called "uncorrelated", or, $(\delta-)$ short range correlated quenched impurities, the system exhibits a spectacular competition between the impurities and gauge effects. The RG equations have a new stable FP of a focal type [76] for the dimensions $D<4$, which exists for symmetry indices $n>1$ and has a physical meaning at the dimensions $D>D_{c}(n)=2(n+36) /(n+18)[76]$. In the impure superconductor, a new focal FP occurs exactly in the domain of symmetry indices $n<365.9$, where the HLM analysis [6] yields a lack of FP for the respective pure system. Having in mind the asymptotic nature of the $\epsilon$-expansions within the RG approach [4,77], one may conclude that this focal FP governs the critical behavior at the real dimension $D=3$ in conventional superconductors $(n=2)$, although the direct substitution of $n=2$ in the above expression for the "lower critical" dimension $D_{c}(n)$ yields $\mathrm{D}_{c}(2)=3.8$. This problem has been further investigated in Ref. [78]. Long-range quenched disorder within the same RG approach to model (1) has been considered in Ref. [79].

The effect of another relevant type of disorder, the socalled "random field disorder", on the phase transitions described by the GL functional (1) has been considered in Ref. [80]. In both short-range and long-range random field correlations $[4,81,82]$ a new stable $\mathrm{FP}$ has been obtained [80]. For short range random correlations this FP is stable below the upper critical dimension $D_{\mathrm{U}}=6$ and for symmetry indices $n>n_{c}=10$. This FP describes a quite specific critical behavior. The situation for long range random correlations is more complicated and here we will advise the reader to follow the discussion in Ref. [80]. In general, the annealed disorder leaves the phase transition properties almost identical to those in 
the pure Abelian-Higgs model (1) whereas the quenched disorder gives lower critical values of the symmetry index $n$, below which the weakly first order phase transition (HLM effect) exists. Thus one may conclude that the quenched disorder acts in the direction of "smearing" the fluctuation-driven first order phase transition, i. e. the HLM effect seems to be much weaker in such systems.

\section{Liquid crystals}

The first theoretical study of the HLM effect on the properties of the nematic-smectic $A$ phase transition in liquid crystals was been performed in Refs. [22,23]. The main result of these investigations is the confirmation of the HLM effect in a close vicinity of this liquid crystal phase transition. Perhaps, because of the liquid crystal anisotropy, which explicitly enters the propagator of the gauge field [23], here the critical value $n_{c}=38.17$ of the symmetry index $n$, below which the weakly first order transition occurs, is lower than in pure superconductors $\left(n_{c}=364.9\right)$ but it is still quite above the real value $n=2$. Thus the RG predictions in Refs. [22,23] in the 1970 s were in favor of a weakly first order phase transition whereas the experimentalists $[83,84]$ at the same time claimed that the same nematic-smectic $A$ phase transition is of second order (for triple and tricritical points in nematic-smectic-A-smectic-C systems, and the interrelationship to the present problem, see Ref. [85]), as well as later papers [86,87]. While in 3D superconductors the HLM effect is very weak and unobservable with available experimental techniques, the size of the same effect in $3 \mathrm{D}$ liquid crystals allows an observation in case of very precise experiment and elimination of obscuring effects as, for example, neighborhood to tricriticality and liquid crystal anisotropy. Experiments $[88,89]$ consistent with the HLM effect has been carried out but, as noticed in Ref [90], these experiments are at their resolution limits and the full implications of the HLM theory remains to be tested. On the other hand, experiments in Ref. [90] demonstrate a discrepancy with the simple approximation ( $\psi=$ const) for the smectic-A filed within the mean-field like approach (Sec. II).

\section{E. Miscellaneous}

Quantum effects. The first account of quantum correlations ("fluctuations") $[4,91,92]$ on the properties of the phase transition to superconducting state has been performed in Ref. [93]. It has been shown [93] that the dynamical critical exponent $z$ produced by the quantum correlations at finite temperatures $(T>0)$ is given by $z=2+18 \epsilon / n$. This is a purely gauge result because, as shown in ref. [94], the critical dynamics of a superconductor in case of neglecting the local gauge effects $(\mathbf{A} \equiv 0)$ is identical to that described by the time dependent GL model (TDGL) in case of lack of conservation laws [95]; a prediction of the latter result has been given for the first time in Ref. [91]. Another study [96] of a gauge model of type (1) is intended to the study of the quantum phase transition $\left(T_{c 0}=0\right)$ in granular superconductors. The simultaneous effect of the local $U(1)$ symmetry, disorder and quantum fluctuations has been considered in studies $[97,98]$ of disordered electronic systems at $T=0$. The problem was further extended to the treatment of quantum phase transitions in underdoped high-temperature superconductors $[99,100]$.

Complex systems. The RG investigation [101] of systems with superconducting and other (non-magnetic) orderings shows that the gauge field $\mathbf{A}(\mathbf{x})$ leads to a drastic modification of the critical behavior in close vicinity of bicritical and tetracritical points (for such points, see, e. g., Ref. $[2,4])$. Near these multicritical points the superconducting fluctuations can be enhanced by the fluctuations of another ordering field and, hence, the HLM effect in such complex systems seems to be stronger than in the simpler case discussed so far [102]. The coupling between the superconducting field $\psi$ and the magnetization mode $\mathbf{M}(\mathbf{x})$ in models of ferromagnetic superconductors intended to describe the coexistence of superconductivity and ferromagnetism in ternary compounds has been investigated by RG in Ref. [103, 104]. It has been shown again that the gauge field $\mathbf{A}(\mathbf{x})$ produces a weakly first order phase transition enhanced by the $M$-fluctuations. The same problem has been further opened in Ref. [105] by a lattice version of the GL model and dual arguments [61] with the conclusion that under certain circumstances the phase transition from the disordered phase to the phase of coexistence of ferromagnetism and superconductivity should be, at least in type II superconductors, of the second order in contrast to the RG prediction $[103,104]$. Another investigation of coupled magnetic and superconducting order parameters, where the gauge effects are also relevant, is intended to describe a state of vortex solid in rear-earth and borocarbide compounds [106, 107].

Unconventional superconductors. Another interesting problem is the behavior of unconventional superconductors $[55,108]$ where the field $\psi$ is a complex vector: $\psi=\left\{\psi_{\alpha}, \alpha=2,3\right\}$, i. e., $n=4$ or 6 , depending on the type of the unconventional Cooper pairing. It has been shown [109], that the HLM FP in this case is unstable towards the parameters, describing the anisotropy of the Cooper pair and the crystal anisotropy, and the new FP points that appear in the theory are unstable for usual values of the symmetry index $(n=4,6)$. It has been shown by analytical calculations [109] that one of the FP points that appear in the RG equations exists for $n>n_{c}=10988$ and is stable for some values above $n_{c}$, at least, in the Hartree limit $(n \rightarrow \infty)$. Owing to these results, and mainly due to a very high value of $n_{c}$, a conclusion has been made that the HLM effect should be more pronounced in unconventional superconductors [109]. The next step along this path of RG studies has been made in Ref. [110], where unconventional superconductors with quenched impurities have been considered. 


\section{V. SHOPOVA, T. E. TSVETKOV, D. I. UZUNOV}

External field effect. In real experiments the external magnetic field can hardly be completely eliminated. Study [38] of the HLM effect in a wider context of nonzero external magnetic field $H_{0}$ shows that the weakly first phase transition discussed so far can be obtained in Landau gauge from a renormalized theory of the equation of state in one loop order; see also Ref. [45]. The phase transition at the second critical magnetic field $H_{c 2}$ has been studied by a $\epsilon=(6-d)$ expansion within the one loop approximation with the conclusion that the magnetic fluctuation effects should produce a fluctuation induced first order phase transition [111]. Further investigations of this problem have been performed in the Hartree limit $(n \rightarrow 0) \quad[112,113]$, and in higher orders of the loop expansion [114,115]; for comments, see Refs. [116,117].

\section{ACKNOWLEDGEMENTS}

Financial support by grants No. P1507 (NFSR, Sofia) and No. G5RT-CT-2002-05077 (EC, SCENET-2, Par$\mathrm{ma}$ ) is acknowledged. D.I.U thanks the hospitality of MPI-PKS (Dresden), where his work on this article was carried out.
[1] V. L. Ginzburg, L. D. Landau, Zh. Eksp. Teor. Fiz. 20, 1064 (1950)

[2] E. M. Lifshitz, L. P. Pitaevskii, Statistical Physics, Part 2, [Landau and Lifshitz Course of Theoretical Physics, vol. 9] (Pergamon Press, Oxford, 1980).

[3] A. A. Abrikosov, Zh. Eksp. Teor. Fiz. 32, 1442 (1957) [Sov. Phys. JETP 5, 1174 (1957)].

[4] D. I. Uzunov, Theory of Critical Phenomena (World Scientific, Singapore, 1993).

[5] J. Zinn-Justin, Quantum Field Theory and Critical Phenomena (Clarendon Press, Oxford, 1993).

[6] B. I. Halperin, T. C. Lubensky, S. K. Ma, Phys. Rev. Lett. 32, 292 (1974).

[7] J-H. Chen, T. C. Lubensky, D. R. Nelson, Phys. Rev. B 17, 4274 (1978).

[8] P. W. Higgs, Phys. Lett. 12, 132 (1964); Phys. Rev. Lett. 13, 508 (1964); Phys. Rev. 145, 1156 (1966).

[9] G. S. Guralnik, C. R. Hagen, T. W. B. Kibble, Phys. Rev. Lett. 13, 585 (1964).

[10] F. Englert, R. Brout, Phys. Rev. Lett. 13, 321 (1964).

[11] T. W. B. Kibble, Phys. Rev. 1955, 1554 (1967).

[12] S. Coleman, Aspects of Symmetry (Cambridge University Press, Cambridge, 1985).

[13] M. Guidry, Gauge Theories (Wiley-VCH Verlag, Weinheim, 2004).

[14] S. Coleman, E. Weinberg, Phys. Rev. D 7 (1973) 1888.

[15] A. D. Linde, Rep. Progr. Phys. 42, 389 (1979).

[16] S. Hikami, Progr. Theor. Phys. 62, 226 (1979).

[17] E. Brézin, C. Itzykson, J. Zinn-Justin, J.-B. Zuber, Phys. Let. B 82, 442 (1979).

[18] A. Vilenkin, E. P. S. Shellard, Cosmic Strings and Other Topological Defects (Cambridge Univesity Press, Cambridge, 1994), Ch. 2.

[19] K. K. Kobayashi, Phys. Lett. A 31, 125 (1970); J. Phys. Soc. Jpn. 29, 101 (1970).

[20] W. L. McMillan, Phys. Rev. A 4, 1238 (1971); 6, 936 (1972).

[21] P. G. de Gennes, Solid State Commun. 10, 753 (1972); Mol. Cryst.Liq. Cryst. 21, 49 (1973).

[22] B. I. Halperin, T. C. Lubensky, Solid State Commun. 14, 994 (1974).

[23] T. C. Lubensky, J.-H. Chen, Phys. Rev. B 17, 366 (1978).

[24] P. G. de Gennes, J. Prost, The Physics of Liquid Crystals, 2nd ed. (Clarendon Press, Oxford, 1993).

[25] S. C. Zhang, T. H. Hansson, S. Kivelson, Phys. Rev.
Lett. 63, 903 (1989).

[26] A. M. J. Schakel, Nucl. Phys. B453 [FS], 705 (1995).

[27] X. G. Wen, Y. S. Wu, Phys. Rev. Lett. 70, 1501 (1993).

[28] L. Pryadko, S. C. Zhang, Phys. Rev. Lett. 73, 3282 (1994).

[29] E. Babaev, A. Sudbo, N. W. Aschroft, Nature 431, 666 (2004).

[30] R. Folk, D. V. Shopova, D. I. Uzunov, Phys. Lett. A 281, 197 (2001).

[31] D. V. Shopova, T. P. Todorov, T. E. Tsvetkov, D. I. Uzunov, Mod. Phys. Lett. B16, 829 (2002).

[32] D. V. Shopova, T. P. Todorov, D. I. Uzunov, Mod. Phys. Lett. B 17, 141 (2003).

[33] D. V. Shopova, T. P. Todorov, J. Phys. Condens. Matter 15, 5783 (2003).

[34] D. V. Shopova, T. P. Todorov, Phys. Lett. A 314, 250 (2003).

[35] , D. V. Shopova, T. P. Todorov, J. Phys. Studies 7, 330 (2003).

[36] T. P. Todorov, PhD Thesis (Bulg. Acad. of Sciences, Sofia, 2003).

[37] R. Folk, Yu. Holovatch, in: Correlations, Coherence, and Order, ed. by D. V. Shopova, D. I. Uzunov (Kluwver Academic/Plenum Publishers, New York, 1999), p. 83. See also, R. Folk, Yu. Holovatch, J. Phys. Stud. 1, 343 (1997).

[38] I. D. Lawrie, J. Phys. C: Solid State Phys. 16, 3513 (1983).

[39] J. C. Rahola, J. Phys. Studies, 5, 304 (2001).

[40] S. W. Lovesey, Z. Physik B: Cond. Matter 40, 117 (1980).

[41] M. Suzuki, D. I. Uzunov, Physica A 204, 702 (1994).

[42] M. Suzuki, D. I. Uzunov, Physica A 216, 489 (1995).

[43] L. Craco, L. De Cesare, I. Rabuffo, I. P. Takov, D. I. Uzunov, Physica A 270, 486 (1999).

[44] O. Madelung (ed.), "Numerical Data and Functional Relationships in Science and Technology", New Series, 21, Superconductors (Springer, Berlin, 1990).

[45] I. D. Lawrie, J. Phys. C: Solid State Phys. 16, 3527 (1983).

[46] R. P. Huebener, Magnetic Flux Structures in Superconductors (Springer, Berlin, 2001).

[47] L. M. Bettencourt, G. J. Stephens, Phys. Rev. B 67, 066105 (2003).

[48] L. M. Abreu, A. P. C. Malbouisson, Phys. Rev. A 322, 111 (2004). 
[49] D. V. Shopova, D. I. Uzunov, Phys. Lett. A 328, 232 (2004).

[50] L. M. Abreu, A. P. C. Malbouisson, Phys. Lett. A 332, 153 (2004) (Corrigendum).

[51] L. M. Abreu, C. de Calan, A. P. C. Malbouisson, condmat/0402053.

[52] L. M. Abreu, A. P. C. Malbouisson, I. Roditi, Eur. Phys. J. 37, 515 (2004).

[53] L. Marotta, M. Camarda, G. G. N. Angilella, F. Siringo, Phys. Rev. B 73, 104517 (2006).

[54] D. I. Uzunov, Phys. Lett. A 87, 11 (1981).

[55] D. I. Uzunov, in: Advances in Theoretical Physics, ed. by E. Caianiello (World Scientific, Singapore, 1990), p. 96; Riken Rev. 2, 9 (1993).

[56] I. D. Lawrie, Nucl. Phys. B 200 [FS4], 1 (1982).

[57] I. D. Lawrie, J. Phys. C: Solid State Phys. 15, L879 (1982).

[58] C. Dasgupta, B. I. Halperin, Phys. Rev. Lett. 47, 1556 (1981).

[59] J. Bartholomew, Phys. Rev. B 28, 5378 (1983).

[60] H. Kleinert, Lett. Al Nuovo Cimento 35, 405 (1982).

[61] M. E. Peshkin, Ann. Phys. (N.Y.) 113, 122 (1978).

[62] M. Kiometzis, H. Kleinert, A. M. J. Schakel, Phys. Rev. Lett 73, 1975 (1994).

[63] M. Kiometzis, H. Kleinert, A. M. J. Schakel, Forschr. Phys. 43, 697 (1995).

[64] I. F. Herbut, J. Phys. A: Math. Gen. 30, 423 (1997).

[65] C. de Calan, F. S. Nogueira, Phys. Rev. B60, 4255 (1999).

[66] P. Olsson, S.Teitel, Phys. Rev. Lett. 80, 1964 (1998).

[67] S. Kolnberger, R. Folk, Phys. Rev. B 41, 4083 (1990).

[68] R. Folk, Yu. Holovatch, J. Phys. A: Math. Gen. 29, 3409 (1996).

[69] I. D. Lawrie, C. Athorne, J. Phys. A: Math. Gen. 16, L587 (1983).

[70] I. D. Lawrie, Phys. Rev. Lett. 78, 979 (1997).

[71] B. Bergerhoff, F. Freire, D. F. Litim, S. Lola, C. Wetterich, Phys. Rev. B 53, 5437 (1996).

[72] I. F. Herbut, Z. Tesanovic, Phys. Rev. Lett. 76, 4588 (1996).

[73] J. A. Hertz, Phys. Rev. B 18, 4875 (1978).

[74] J. A. Hertz, Phys. Scripta T10, 1 (1985).

[75] D. Boyanovsky, J. K. Cardy, Phys. Rev. B 25, 7058 (1982).

[76] D. I. Uzunov, E. R. Korutcheva, Y. T. Millev, J. Phys. A: Math. Gen., 16, 247 (1983); 17, 247 (1984); Err., ibid, 17, 1775 (1984).

[77] I. D. Lawrie, Y. T. Millev, D. I. Uzunov, J. Phys. A: Math. Gen. 20, 1599 (1987); (Err.) ibid, 20, 6159 (1987).

[78] C. Athorne, I. D. Lawrie, Nucl. Phys. B 257 [FS14], 577 (1985).

[79] E. R. Korutcheva, Y. T. Millev, J. Phys. A: Math. Gen., 17, L511 (1984).

[80] G. Busiello, L. De Cesare, D. I. Uzunov, Phys. Rev. B 34, 4932 (1986).

[81] D. I. Uzunov, E. R. Korutcheva, Y. T. Millev, Physica A 129, 535 (1985).

[82] D. I. Uzunov, E. R. Korutcheva, Y. T. Millev, Phys. Status Solidi (b) 130, 243 (1985).

[83] D. Davidov, C. R. Safinya, M. Kaplan, S. S. Dana, R. Schaetzing, R. J. Birgeneau, J. D. Litster, Phys. Rev. B 19, 1657 (1979).
[84] J. D. Litster, R. J. Birgeneau, M. Kaplan, C. R. Safinya, in: Order in Strongly Fluctuating Condensed Matter Systems, ed. by T. Riste (Plenum, New York, 1980).

[85] C. C. Huang, S. C. Lien, Phsy. Rev. Lett. 47, 1917 (1981).

[86] C. W. Garland, G. Nounesis, Phys. Rev. E 49, 2964 (1994).

[87] I. Lelidis, G. Durand, Phys. Rev. Lett. 73, 672 (1994).

[88] M. A. Anisimov, V. P. Voronov, E. E. Gorodetskii, V. E. Podneks, F. Kholmudorov, Pisma Zh. Eksp. Teor. Fiz. 45, 336 (1987) [JETP Lett. 45, 425 (1987)].

[89] M. A. Anisimov, P. E. Cladis, E. E. Gorodetskii, D. A. Huse, V. E. Podneks, V. G. Taratuta, W. van Saarloos, V. P. Voronov, Phys. Rev. A 41, 6749 (1990).

[90] A. Yethiraj, J. Bechhoefer, Phys. Rev. Lett. 84, 3642 (2000).

[91] J. A. Hertz, Phys. Rev. B 14, 1165 (1976).

[92] D. V. Shopova, D. I. Uzunov, Phys. Rep. C 379, 1 (2003).

[93] M. K. Bushev, D. I. Uzunov, Phys. Lett. A 76, 306 (1980); Err., ibid, 78A, 491 (1980).

[94] D. I. Uzunov, Phys. Lett. A 78, 395 (1980).

[95] P. C. Hohenberg, B. I. Halperin, Rev. Mod. Phys.49, 435 (1977).

[96] M. P. A. Fisher, G. Grinstein, Phys. Rev. Lett. 60, 208 (1988).

[97] M. P. A. Fisher, G. Grinstein, S. M. Girvin, Phys. Rev. Lett. 64, 587 (1990).

[98] I. F. Herbut, Phys. Rev. B 57, 13729 (1998).

[99] D. Matthey, N. Reyren, J.-M. Triscone, T. Schneider, arXiv:cond-mat/0603079.

[100] T. Schneider, cond-mat/0610230030.

[101] N. S. Tochev, D. I. Uzunov, J. Phys. A: Math. Gen. 14, 521 (1981).

[102] N. S. Tochev, D. I. Uzunov, J. Phys. A: Math. Gen. 14, L103 (1981).

[103] N. Grewe, B. Schuh, Z. Phys. B 36, 89 (1979).

[104] N. Grewe, B. Schuh, Phys. Rev. B 22, 3183 (1980).

[105] P. Bhattacharyya, J. Phys. C: Solid State Phys. 16, L1011 (1983).

[106] L. Radzihovsky, A. M. Ettouhami, K. Saunders, J. Toner, Phys. Rev. Lett. 87, 027001 (2001).

[107] A. M. Ettouhami, K. Saunders, L. Radzihovsky, J. Toner, Phys. Rev. B 71, 224506 (2005).

[108] E. J. Blagoeva, G. Busiello, L. De Cesare, Y. T. Millev, I. Rabuffo, D. I. Uzunov, Phys. Rev. B 42, 287 (1990).

[109] Y. T. Millev, D. I. Uzunov, Phys. Lett. A 145, 287 (1990).

[110] G. Busiello, L. De Cesare, Y. T. Millev, I. Rabuffo, D. I. Uzunov, Phys. Rev. B 43, 1150 (1991).

[111] E. Brezin, D. R. Nelson, A. Thiaville, Phys. Rev. B 11 (1985) 7124

[112] I. Affleck, E. Brésin, Nucl. Phys. B 257 [FS14], 451 (1985).

[113] L. Radzihovsky, Phys. Rev. Lett. 74, 4722 (1995).

[114] E. Brézin, A. Fijita, S. Hikami, Phys. Rev. Lett. 65, 1949 (1990).

[115] G. P. Mikitik, Zh. Eksp. Teor. Fiz. 101, 1042 (1992) [Sov. Phys. JETP 74, 558 (1992)].

[116] L. Radzihovsky, Phys. Rev. Lett. 76, 4451 (1996).

[117] I. F. Herbut, Z. Tesanovic, Phys. Rev. Lett. 76, 4450 (1996). 


\section{ВПЛИВ КАЛІБРУВАЛЬНИХ ЕФЕКТІВ НА ФАЗОВІ ПЕРЕХОДИ В НАДПРОВІДНИКАХ}

Д. В. Шопова ${ }^{1}$, Т. Е. Цветков ${ }^{1}$, Д. І. Узунов ${ }^{1,2}$

${ }^{1}$ Інститут фізики твердого тіла, СР лабораторіл, Болгарсъка АН $B G-1784$, м. Собія, Болгарія

${ }^{2}$ Інститут складних систем імені Макса Планка

Нъотнічер-штрассе, 38, 01187, м. Дрезден, Німеччина

Здійснено огляд класичних і сучасних результатів, які стосуються впливу калібрувальних ефектів на властивості фазового переходу в надпровідний стан в об'ємних і плівкових надпровідниках. Також обговорено подібні проблеми в описі інших природних систем (рідкі кристали, квантова теорія поля, ранній Всесвіт). Детально розглянуто відносно сильний вплив калібрувальних ефектів на флюктуації поля впорядкування при малих вимірностях простору $D$ i, зокрема, в тонких (квазідвовимірних) плівках. Особливу увагу звернуто на флюктуацї калібрувального поля. Показано, що механізм впливу цих калібрувальних флюктуацій на порядок та інші властивості фазового переходу змінюється зі зміною просторової вимірности $D$. Обговорено проблему експериментального підтвердження теоретичних передбачень щодо порядку фазового переходу в калібрувальних системах. 